\title{
A Mesoporous Indium Metal-Organic Framework: Remarkable Advances in Catalytic Activity for Strecker Reaction of Ketones.
}

Daniel Reinares-Fisac, Lina María Aguirre-Díaz, Marta Iglesias, Natalia Snejko, Enrique Gutiérrez-Puebla, M. Ángeles Monge, ${ }^{*}$ Felipe Gándara*

\section{Supporting material}

S1. Experimental procedure

- General

- $\quad$ Synthesis of InPF-110

- $\quad$ Catalytic activity experiments procedure

S2. Single crystal X-ray diffraction and structure of InPF-110 and InPF-110 aniline

S3. Characterization of InPF-110

S4. Single crystal X-ray diffraction and structure of InPF-50

S5. Catalytic activity experiments background

S6. Catalytic activity data

S-10

S7. Recycle reactions

S8. Characterization of the Strecker $3 \mathrm{C}$ reaction products

S9. Spectra for characterized compounds

S-18

S10. References

S-36 


\section{- S1. EXPERIMENTAL PROCEDURE}

\subsection{General}

All reagents and solvents employed were commercially available and used as received without further purification except for $\ln \left(\mathrm{NO}_{3}\right)_{3}$, which was previously dehydrated at $105{ }^{\circ} \mathrm{C}$ overnight: In $\left(\mathrm{NO}_{3}\right)_{3} \cdot \mathrm{xH}_{2} \mathrm{O}$ (99.999\%-In PURATREM from Strem Chemicals); 1,3,5-tris(4carboxyphenyl)benzene, $\mathrm{H}_{3}$ btb (purchased from TCl Europe); N,N-dimethylformamide (DMF; from Scharlab) and nitric acid $69.5 \%$ reagent grade (from Scharlab). NMR spectra were recorded in an AVIII-HD Nanobay AMX-300 equipment using $\mathrm{CDCl}_{3}$ as solvent. IR spectra were recorded from $\mathrm{KBr}$ pellets in the range $4000-400 \mathrm{~cm}^{-1}$ on a Nicolet FT-IR 20SXC spectrometer. Thermogravimetric and differential thermal analysis (TGA-DTA) was performed using a SDT Q600 from TA Instruments equipment in a temperature range between 30 and $800{ }^{\circ} \mathrm{C}$ in air $\left(100 \mathrm{~mL} / \mathrm{min}\right.$ flow) atmosphere and heating rate of $10^{\circ} \mathrm{C} / \mathrm{min}$. A CNHS PERKIN ELMER 2400 analyzer was employed for the elemental analysis. Powder X-ray diffraction (PXRD) patterns were measured with a Bruker D8 diffractometer with a copper source operated at $1600 \mathrm{~W}$, with step size $=0.02^{\circ}$ and exposure time $=0.5 \mathrm{~s} / \mathrm{step}$. PXRD measurements were used to check the purity of the obtained microcrystalline products by a comparison of the experimental results with the simulated patterns obtained from single-crystal $\mathrm{X}$-ray diffraction data. $\mathrm{N}_{2}$ sorption isotherms were measured with an AUTOSORB-1 from Quantachrome Instruments at 77K in the relative pressure range of $10^{-2}$ to 1 . Before adsorption measurements, the samples were activated and outgassed at $473 \mathrm{~K}$ overnight. Exact mass measurements were performed in a VARIAN AutoSpec equipment employing "Fast Atom Bombardement" (FAB) method.

\subsection{Preparation of InPF-110}

$\left[\ln { }_{3} \mathrm{O}(\mathrm{btb})_{2}\left(\mathrm{HCO}_{2}\right)(\mathrm{L})\right]$ : In a Teflon vessel, $99 \mathrm{mg}$ of $\operatorname{In}\left(\mathrm{NO}_{3}\right)_{3} \cdot \mathrm{xH}_{2} \mathrm{O}$ previously dried at $105^{\circ} \mathrm{C}$ overnight $(0,33 \mathrm{mmol})$ and $66 \mathrm{mg}$ of 1,3,5-tris(4-carboxyphenyl)benzene $\left(\mathrm{H}_{3} \mathrm{btb} ; 0.15 \mathrm{mmol}\right)$ were added. Later, we dissolved them in $2.5 \mathrm{~mL}$ of N,N-dimethylformamide (DMF) and $0.8 \mathrm{~mL}$ of nitric acid were added. Next, the vessel was sealed and placed in a preheated oven at $150^{\circ} \mathrm{C}$ for a time of 4 hours and cooled to room temperature. Pure, white, needle-like crystals were obtained after being centrifuged and washed with DMF and methanol, ethanol and/or water. Powder X-ray diffraction supported the purity of the sample. Yield: $0.059 \mathrm{~g}, 58 \%$. Elemental analysis, found (calculated): C, 50.19 (51.12); H, $2.8(2.42)$. IR (KBr, cm$\left.{ }^{-1}\right): 3439 v(\mathrm{O}-\mathrm{H}), 1613$ and $1585 v(\mathrm{C}=\mathrm{O}), 1527 v(\mathrm{C}-\mathrm{O}), 1404 v(\mathrm{C}-\mathrm{C}), 1178,1109,1013,856$ and $700 v(\mathrm{C}-\mathrm{C})$ aromatic, $783 v(\mathrm{C}-\mathrm{C}), 497$ and $396 v(\ln -\mathrm{O})$.

\subsection{Catalytic activity experiments procedure}

Catalyst was activated by heating at $200^{\circ} \mathrm{C}$ for a period of 24 hours after soaking three days in the corresponding solvent (water, ethanol or methanol), which was changed three times per day. Activation temperature was chosen from TG data in order to remove all traces of synthesis solvent inside the pore. Then, the mixture of ketone, amine and trimethylsilyl cyanide (TMSCN) was added to a Schlenk tube $(0.85 \mathrm{mmol}$ of ketone, $0.77 \mathrm{mmol}$ of amine and $0.85 \mathrm{mmol}$ of TMSCN), where the MOF has been previously introduced. The $10 \%$ of excess of ketone in relation to amine is used as a pattern in ${ }^{1} \mathrm{H}$ NMR. The mixture (in solvent-free conditions) was stirred $(300 \mathrm{rpm})$ at $25^{\circ} \mathrm{C}$ between 0.85 and $48 \mathrm{~h}$ depending on the substrate, under $\mathrm{N}_{2}$ atmosphere. For comparative purpose, the same standard reaction conditions were used for all tested substrates, and therefore the reaction parameters were not further optimized for each one of them. The completion of the reaction was monitored by gas chromatography and ${ }^{1} \mathrm{H}$ NMR. When reaction was completed and samples were taken, the content of the Schlenk tube was mixed with DCM in order to dissolve the Strecker $\alpha$-aminonitrile and recover the catalyst by 
filtration. X-ray diffraction patterns were obtained before and after each experiment to ensure crystallinity, purity and recyclability of the MOF.

Catalyst was reused at least in 10 cycles. In each case, after recovery, InPF-110 was washed with methanol and dried overnight following the same procedure described above, without significant loss of activity (yield of $90 \%$ in the tenth run).

The leaching test was performed. The mixture remained 5 minutes within the conditions previously described. After that, part of the liquid was separated and transferred to an empty Schlenk. After 24 hours, different products were found in the mixture, mainly composed of unreacted acetophenone (24\%) and aniline (12\%), a small amount of aminonitrile (22\%), and other undetermined products (scheme 1 shows the main possible products). This probed that the presence of the catalyst is necessary in order to selectively complete the organic transformation.

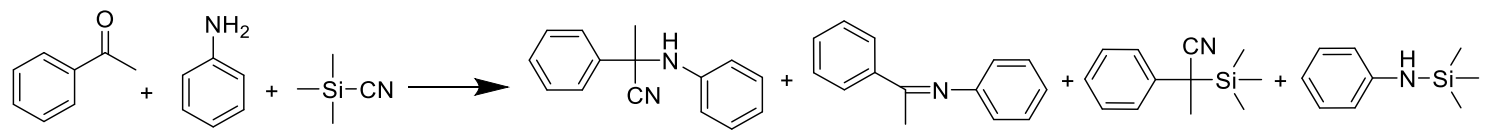

Scheme S1 Strecker reaction and possible by-products: $\alpha$-aminonitrile, imine, cyanohdrine and silanamine. 


\section{- S2. X-RAY STRUCTURE DETERMINATION}

\subsection{Main crystallographic data for InPF-110 and InPF-110aniline}

Table S1 and S2 summarizes the main crystal and refinement data for InPF-110 and InPF-110 aniline respectively. Crystals were selected under a polarizing optical microscope for a singlecrystal X-ray diffraction experiment. Single-crystal X-ray data were obtained in a Bruker four circle kappa-diffractometer equipped with a Cu INCOATED microsource, operated at $30 \mathrm{~W}$ power $(45 \mathrm{kV}, 0.60 \mathrm{~mA})$ to generate $\mathrm{Cu}$ Ka radiation $(\lambda=1.54178 \AA)$, and a Bruker VANTEC 500 area detector (microgap technology). Diffraction data were collected exploring over a hemisphere of the reciprocal space in a combination of $\varphi$ and $\omega$ scans to reach a resolution of $0.85 \AA$, using a Bruker APEX $2^{1}$ software suite (each exposure, depending on $\omega$, was of 56,90 or $140 \mathrm{~s}$ covering $1^{\circ}$ in $\omega$ or $\varphi$ ). Unit cell dimensions were determined for least-squares fit of reflections with $I>4 \sigma$. The structures were solved by intrinsic phase methods ${ }^{2}$. The hydrogen atoms were fixed at their calculated positions using distances and angle constraints. All calculations were performed using APEX2 software for data collection and OLEX2-1.2 $2^{3}$ and SHELXTL ${ }^{4}$ to resolve and refine the structure.

Table S2.1 Main crystallographic data for InPF-110 compound. Data recovered from InPF-110 crystal after evacuation.

\begin{tabular}{|c|c|}
\hline Identification code & InPF-110 \\
\hline Empirical formula & $\mathrm{C}_{168} \mathrm{H}_{90} \mathrm{In}_{9} \mathrm{O}_{48}$ \\
\hline Formula weight & 3909.77 \\
\hline Temperature/K & 250.0 \\
\hline Crystal system & hexagonal \\
\hline Space group & P-62c \\
\hline $\mathbf{a} / \AA$ & $31.8903(7)$ \\
\hline $\mathbf{b} / \mathbf{A}$ & $31.8903(7)$ \\
\hline $\mathbf{c} / \AA$ & 17.1915(5) \\
\hline $\mathbf{\alpha} / /^{\circ}$ & 90 \\
\hline$\beta /^{\circ}$ & 90 \\
\hline $\mathrm{y} /{ }^{\circ}$ & 120 \\
\hline Volume $/ \AA^{3}$ & 15141.2(8) \\
\hline $\mathbf{Z}$ & 2 \\
\hline$\rho_{\text {calc }} \mathbf{g} / \mathrm{cm}^{3}$ & 0.858 \\
\hline$\mu / \mathrm{mm}^{-1}$ & 5.743 \\
\hline$F(000)$ & 3846.0 \\
\hline Crystal size $/ \mathrm{mm}^{3}$ & $0.2 \times 0.04 \times 0.04$ \\
\hline Radiation & CuKa $(\lambda=1.54178)$ \\
\hline 20 range for data collection $/^{\circ}$ & 3.198 to 130.156 \\
\hline Index ranges & $-37 \leq \mathrm{h} \leq 33,-30 \leq \mathrm{k} \leq 37,-19 \leq \mathrm{I} \leq 20$ \\
\hline Reflections collected & 103782 \\
\hline Independent reflections & $8925\left[R_{\text {int }}=0.2007, R_{\text {sigma }}=0.0987\right]$ \\
\hline Data/restraints/parameters & $8925 / 0 / 360$ \\
\hline Goodness-of-fit on $F^{2}$ & 1.006 \\
\hline Final $R$ indexes [l>=2 $\sigma(\mathrm{I})]$ & $R_{1}=0.0961, w R_{2}=0.2409$ \\
\hline Final $\mathbf{R}$ indexes [all data] & $R_{1}=0.1607, w R_{2}=0.3034$ \\
\hline Largest diff. peak/hole / e $\AA^{-3}$ & $1.21 /-2.10$ \\
\hline Flack parameter & $-0.010(8)$ \\
\hline
\end{tabular}


Table S2.2 Main crystallographic data for InPF-110 with coordinated aniline.

\begin{tabular}{|c|c|}
\hline Identification code & InPF-110 Aniline \\
\hline Empirical formula & $\mathrm{C}_{188} \mathrm{H}_{97.5} \mathrm{In}_{9} \mathrm{~N}_{1.5} \mathrm{O}_{46.5}$ \\
\hline Formula weight & 4154.55 \\
\hline Temperature/K & 250.0 \\
\hline Crystal system & hexagonal \\
\hline Space group & P-62c \\
\hline $\mathbf{a} / \check{A}$ & $31.7180(15)$ \\
\hline $\mathbf{b} / \mathbf{A}$ & $31.7180(15)$ \\
\hline $\mathbf{c} / \ddot{\mathbf{A}}$ & $17.1352(9)$ \\
\hline$\alpha / /^{\circ}$ & 90 \\
\hline$\beta /^{\circ}$ & 90 \\
\hline $\mathrm{y} /^{\circ}$ & 120 \\
\hline Volume $/ \AA^{3}$ & 14929.0(16) \\
\hline $\mathbf{Z}$ & 2 \\
\hline$\rho_{\text {calc }} \mathbf{g} / \mathrm{cm}^{3}$ & 0.924 \\
\hline$\mu / \mathrm{mm}^{-1}$ & 5.846 \\
\hline$F(000)$ & 4098.0 \\
\hline Crystal size $/ \mathrm{mm}^{3}$ & $0.2 \times 0.04 \times 0.04$ \\
\hline Radiation & CuKa $(\lambda=1.54178)$ \\
\hline 20 range for data collection $/{ }^{\circ}$ & 3.216 to 130.4 \\
\hline Index ranges & $-37 \leq \mathrm{h} \leq 37,-37 \leq \mathrm{k} \leq 37,-19 \leq \mathrm{I} \leq 19$ \\
\hline Reflections collected & 119004 \\
\hline Independent reflections & $8819\left[R_{\text {int }}=0.1249, R_{\text {sigma }}=0.0468\right]$ \\
\hline Data/restraints/parameters & $8819 / 12 / 420$ \\
\hline Goodness-of-fit on $F^{2}$ & 1.140 \\
\hline Final $R$ indexes [l>=2 $\sigma(\mathrm{I})]$ & $\mathrm{R}_{1}=0.0649, \mathrm{wR}_{2}=0.1825$ \\
\hline Final $\mathbf{R}$ indexes [all data] & $R_{1}=0.0777, w R_{2}=0.1939$ \\
\hline Largest diff. peak/hole / e Ă ${ }^{-3}$ & $0.74 /-1.18$ \\
\hline Flack parameter & $0.114(6)$ \\
\hline
\end{tabular}


Formula [ $\left.\mathrm{In}_{3} \mathrm{O}\left(\mathrm{HCO}_{2}\right)(\mathrm{btb})_{2}(\mathrm{~L})\right]$; InPF-110 CCDC number 1483220; InPF-110 with aniline CCDC number 1483222. Formula weight $1276.27+\mathrm{L}(\mathrm{L}=$ methanol, ethanol, water)

\subsection{IR spectra $\left(\mathrm{KBr} ; \mathrm{cm}^{-1}\right)$}

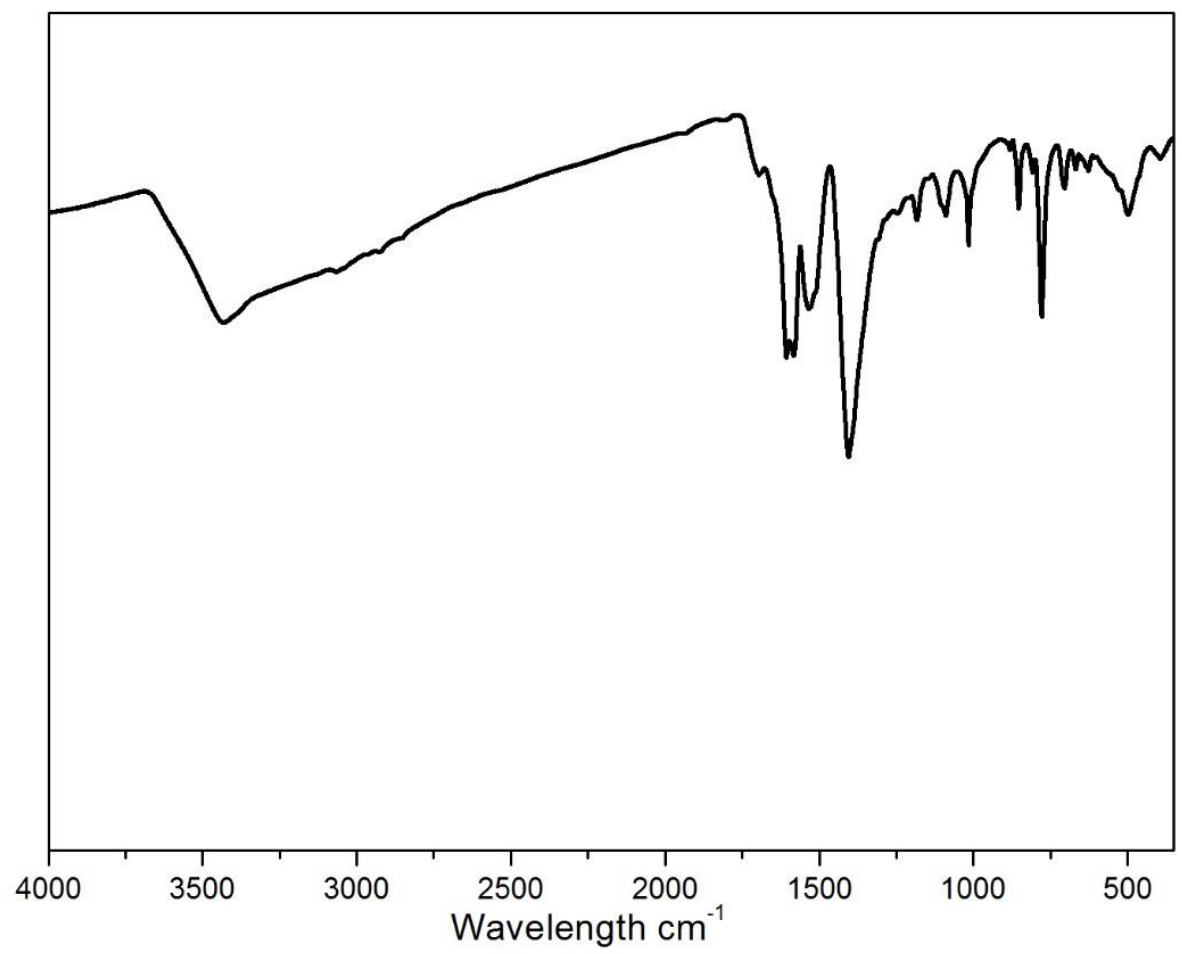

Figure S3.1 Infrared transmittance spectra of MOF InPF-110.

\subsection{Thermogravimetric analysis (TGA)}

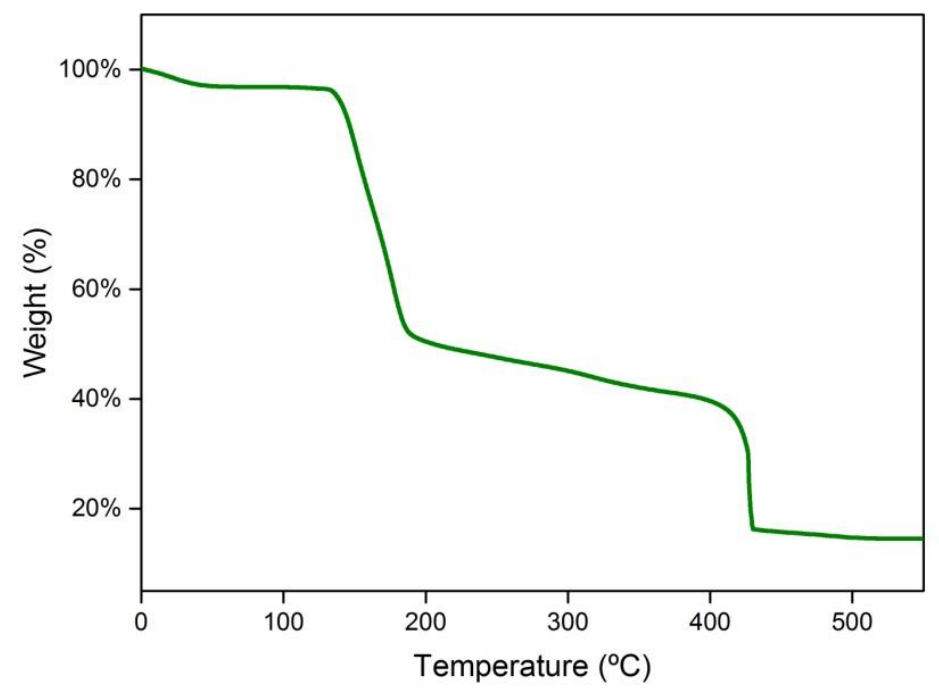

Figure S3.2a TG-DTA curve for as-synthesized InPF-110. Pores are filled with DMF molecules, which evacuates from 150 to $200^{\circ} \mathrm{C}$, corresponding to a loss of $45 \%$ of the total weight. The structure finally collapses when temperature reaches $400^{\circ} \mathrm{C}$. The final residue is $\ln _{2} \mathrm{O}_{3}$. 


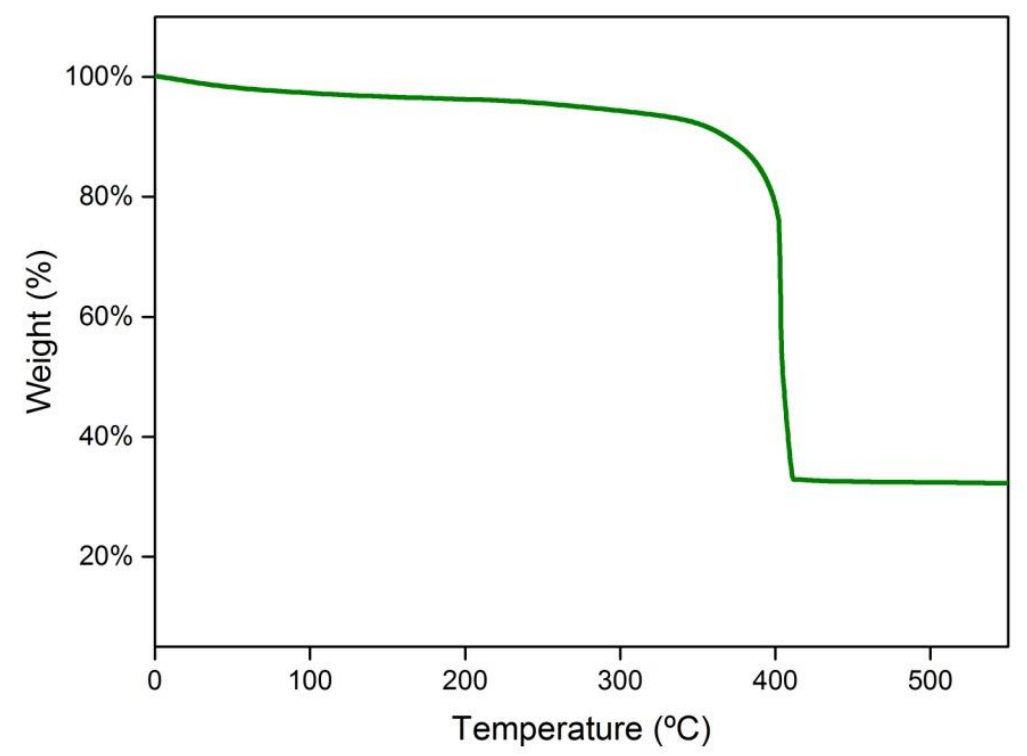

Figure S3.2b TG-DTA curve for activated InPF-110. The structure collapses after $400{ }^{\circ} \mathrm{C}$. A loss of $5 \%$ occurs between room temperature and $300{ }^{\circ} \mathrm{C}$ due to water and/or methanol present in pore.

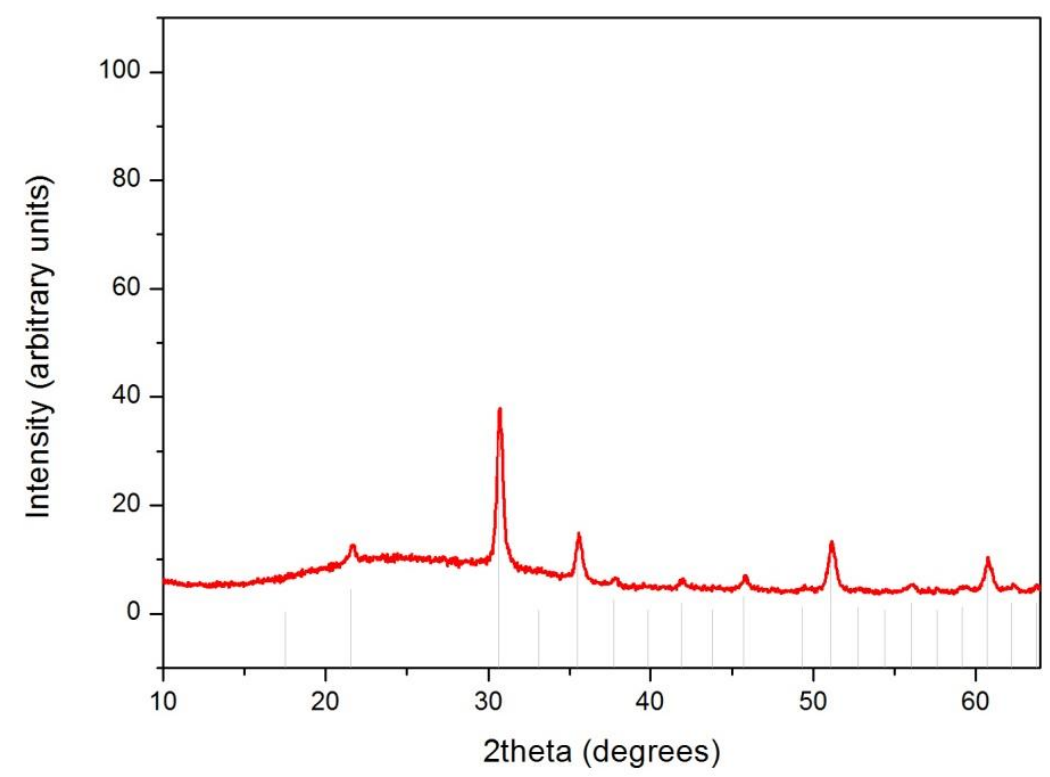

Figure S3.2c Powder X-ray diffraction of residues after thermogravimetric analysis, fitting according to $\ln _{2} \mathrm{O}_{3}$ PXRD ICSD pattern $640179 .^{5}$ 
- S4. SINGLE CRYSTAL X-RAY DIFFRACTION AND STRUCTURE OF InPF-50

Table S4 Main crystallographic data for InPF-50. CCDC number 1483221.

\begin{tabular}{|c|c|}
\hline Identification code & InPF50 \\
\hline Empirical formula & $\mathrm{C}_{30} \mathrm{H}_{18} \ln \mathrm{NO}_{10.5}$ \\
\hline Formula weight & 675.27 \\
\hline Temperature/K & 250 \\
\hline Crystal system & orthorhombic \\
\hline Space group & Pbcn \\
\hline $\mathbf{a} / \ddot{A}$ & $17.5574(8)$ \\
\hline b/Ä & 20.8944(9) \\
\hline c/Ä & $16.8168(8)$ \\
\hline$\alpha /^{\circ}$ & 90 \\
\hline$\beta /^{\circ}$ & 90 \\
\hline $\mathrm{y} /{ }^{\circ}$ & 90 \\
\hline Volume $/ \AA^{3}$ & $6169.3(5)$ \\
\hline $\mathbf{Z}$ & 8 \\
\hline$\rho_{\text {calc }} \mathrm{g} / \mathrm{cm}^{3}$ & 1.454 \\
\hline$\mu / \mathrm{mm}^{-1}$ & 6.623 \\
\hline$F(000)$ & 2704.0 \\
\hline Crystal size $/ \mathrm{mm}^{3}$ & $0.175 \times 0.175 \times 0.075$ \\
\hline Radiation & CuKa $(\lambda=1.54178)$ \\
\hline $2 \Theta$ range for data collection $/{ }^{\circ}$ & 6.576 to 130.532 \\
\hline Index ranges & $-20 \leq \mathrm{h} \leq 20,-24 \leq \mathrm{k} \leq 23,-19 \leq \mathrm{I} \leq 19$ \\
\hline Reflections collected & 30660 \\
\hline Independent reflections & $5195\left[R_{\text {int }}=0.0600, R_{\text {sigma }}=0.0389\right]$ \\
\hline Data/restraints/parameters & $5195 / 0 / 378$ \\
\hline Goodness-of-fit on $F^{2}$ & 1.158 \\
\hline Final $R$ indexes [l>=2 $\sigma(\mathrm{I})]$ & $R_{1}=0.0866, w R_{2}=0.2445$ \\
\hline Final $\mathbf{R}$ indexes [all data] & $R_{1}=0.1008, w R_{2}=0.2618$ \\
\hline Largest diff. peak/hole / e $\AA^{-3}$ & $1.17 /-1.23$ \\
\hline
\end{tabular}




\section{- S5. CATALYTIC ACTIVITY EXPERIMENTS BACKGROUND}

Table S5.1 Catalytic activity of several homogeneous and heterogeneous catalysts used in the acetophenone mediated 3C-Strecker reaction.

\begin{tabular}{|c|c|c|c|c|}
\hline Catalyst & Type & $\begin{array}{l}\text { Reaction } \\
\text { conditions }\end{array}$ & $\begin{array}{l}\text { Time and } \\
\text { yield }\end{array}$ & Reference \\
\hline o-Benzenedisulfonimide & homogeneous & $\begin{array}{l}2-5 \mathrm{~mol} \%, 20- \\
25=\mathrm{C}\end{array}$ & $\begin{array}{l}5 \min -6 \mathrm{~h} \\
93-95 \%\end{array}$ & Ghigo et al. ${ }^{6}$ \\
\hline $\begin{array}{l}\text { Sulfonic acid based } \\
\text { nanoreactors }\end{array}$ & heterogeneous & $\begin{array}{l}5 \text { mol\%, r.t., } \\
\text { solvent-free }\end{array}$ & $55 \min ; 98 \%$ & Karimi et al. ${ }^{7}$ \\
\hline Nafion-Fe & heterogeneous & $\begin{array}{l}100 \mathrm{mg}, 80^{\circ} \mathrm{C}, \\
\mathrm{DCM}\end{array}$ & $6 \mathrm{~h} ; 62 \%$ & Mathew et al. ${ }^{8}$ \\
\hline $\mathrm{Ga}(\mathrm{OTf})_{3}$ & heterogeneous & $\begin{array}{l}5 \mathrm{~mol} \% \text {, r.t., } \\
\text { DCM }\end{array}$ & 5 h; $98 \%$ & Olah et al. ${ }^{9}$ \\
\hline $\begin{array}{l}\text { BINOL-derives } \\
\text { phosphoric acids }\end{array}$ & homogeneous & $\begin{array}{l}10 \mathrm{~mol} \% \\
40^{\circ} \mathrm{C} \text {, toluene }\end{array}$ & $\begin{array}{l}24-48 \text { h; } 98 \\
\%\end{array}$ & $\begin{array}{l}\text { Jun-An Ma et } \\
\text { al. } .^{10}\end{array}$ \\
\hline $\operatorname{lnBr}_{3}$ & homogeneous & $\begin{array}{l}10 \text { mol\%, up to } \\
80^{\circ} \mathrm{C} \text {, toluene }\end{array}$ & 27 h; $96 \%$ & Sakai et al. ${ }^{11}$ \\
\hline
\end{tabular}

Table S5.2 Catalytic activity of different $p$-MOFs in the 3C-Strecker reaction with acetophenone, aniline and trimethylsilyl cyanide.

\begin{tabular}{|c|c|c|c|c|}
\hline Catalyst & Type & $\begin{array}{l}\text { Reaction } \\
\text { conditions }\end{array}$ & $\begin{array}{l}\text { Time and } \\
\text { yield }\end{array}$ & Reference \\
\hline In-MOF 1 & heterogeneous & $\begin{array}{l}50 \text { mol\%, r.t., } \\
\mathrm{CDCl}_{3}(2 \mathrm{C})\end{array}$ & $96 \mathrm{~h} ; 46.5 \%$ & $\begin{array}{l}\text { Jifu Zheng et } \\
\text { al. }{ }^{12}\end{array}$ \\
\hline In-MOF 2 & heterogeneous & $\begin{array}{l}50 \text { mol\%, r.t., } \\
\mathrm{CDCl}_{3}(2 \mathrm{C})\end{array}$ & 96 h; $99.6 \%$ & $\begin{array}{l}\text { Jifu Zheng et } \\
\text { al. }^{12}\end{array}$ \\
\hline InGaPF-1 & heterogeneous & $\begin{array}{l}1 \text { mol\%, r.t., } \\
\text { Solvent-free }\end{array}$ & $0.42 \mathrm{~h} ; 50 \%$ & Monge et al. ${ }^{13}$ \\
\hline InGaPF-2 & heterogeneous & $\begin{array}{l}1 \text { mol\%, r.t., } \\
\text { Solvent-free }\end{array}$ & $0.75 \mathrm{~h} ; 67 \%$ & Monge et al. ${ }^{13}$ \\
\hline InGaPF-3 & heterogeneous & $\begin{array}{l}1 \text { mol\%, r.t., } \\
\text { Solvent-free }\end{array}$ & 2 h; $80 \%$ & Monge et al. ${ }^{13}$ \\
\hline InPF-11 $\alpha$ & heterogeneous & $\begin{array}{l}2.5 \mathrm{~mol} \%, 80^{\circ} \mathrm{C}, \\
\text { Solvent-free }\end{array}$ & $4 \mathrm{~h} ; 65 \%$ & Monge et al. ${ }^{13}$ \\
\hline InPF-11 $\beta$ & heterogeneous & $\begin{array}{l}1 \text { mol\%, r.t., } \\
\text { Solvent-free }\end{array}$ & $0.67 \mathrm{~h} ; 67 \%$ & Monge et al. ${ }^{13}$ \\
\hline GaPF-1 & heterogeneous & $\begin{array}{l}1 \text { mol\%, r.t., } \\
\text { Solvent-free }\end{array}$ & $0.63 \mathrm{~h} ; 67 \%$ & Monge et al. ${ }^{13}$ \\
\hline AIPF-1 & heterogeneous & $\begin{array}{l}1 \text { mol\%, r.t., } \\
\text { Solvent-free }\end{array}$ & 8 h; $75 \%$ & Monge et al. ${ }^{13}$ \\
\hline
\end{tabular}


- S6. CATALYTIC ACTIVITY DATA

Table S6 Screening of catalyst loadings and scaled up in the Strecker one-pot three component reaction using acetophenone, aniline and TMSCN (1.1:1:1.1).

\begin{tabular}{ccccc}
$\begin{array}{c}\text { Catalyst loading } \\
(\mathbf{m o l} \%)^{a}\end{array}$ & $\begin{array}{c}\text { Time } \\
(\mathbf{h})\end{array}$ & $\begin{array}{c}\text { InPF-110 } \\
\text { [catalyst loading] }\end{array}$ \\
\cline { 3 - 5 } & 0.1 & $6.5 \mathrm{~h}$ & $90 \%$ & 900 \\
2 & 0.25 & $5 \mathrm{~h}$ & $96 \%$ & 384 \\
3 & 0.5 & $4 \mathrm{~h}$ & $99 \%$ & 198 \\
4 & 1 & $2.9 \mathrm{~h}$ & $99 \%$ & 99 \\
5 & 2 & $2.6 \mathrm{~h}$ & $99 \%$ & 49.5 \\
6 & $0.5^{b}$ & $5 \mathrm{~h}$ & $98 \%$ & 196 \\
\hline
\end{tabular}

${ }^{a}$ Reaction conditions: acetophenone, amine, TMSCN (1.1:1:1.1), 0.5 mol\% catalyst based on $\left[\ln _{3} \mathrm{O}(\mathrm{btb})_{2}\left(\mathrm{HCO}_{2}\right)(\mathrm{L})\right], \mathrm{N}_{2}$ atmosphere, $25^{\circ} \mathrm{C}$; catalyst washed with $\mathrm{L}=$ methanol. ${ }^{b}$ Scaled up to 5 times. ${ }^{c}$ Yield by ${ }^{1} \mathrm{H}$ NMR. ${ }^{d} \mathrm{TON}=(\mathrm{mmol}$ substrate $/ \mathrm{mmol}$ catalyst $)$

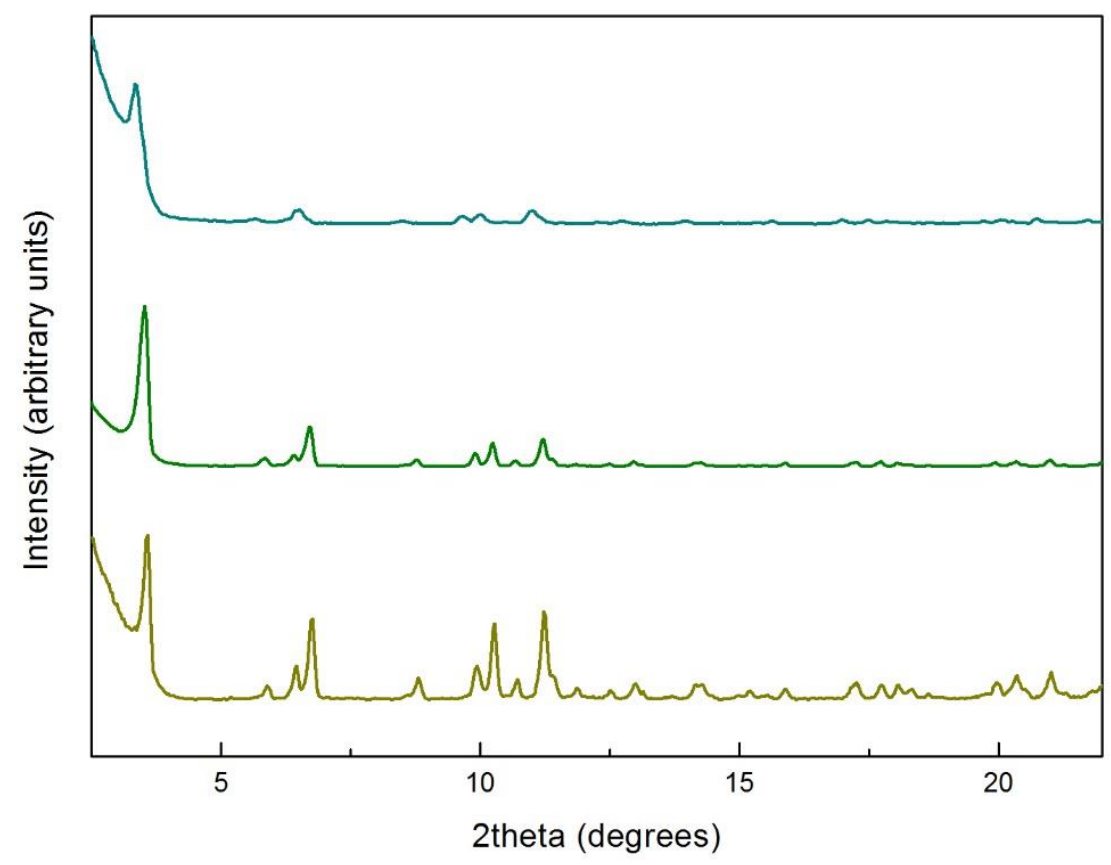

Figure S6.1 Bottom up, InPF-110 as-synthesized, activated and after standard catalytic reaction. 
- S7. RECYCLE REACTIONS

Table S7 Strecker one-pot three component reaction using acetophenone, aniline and TMSCN (1.1:1:1.1) up to 10 cycles.

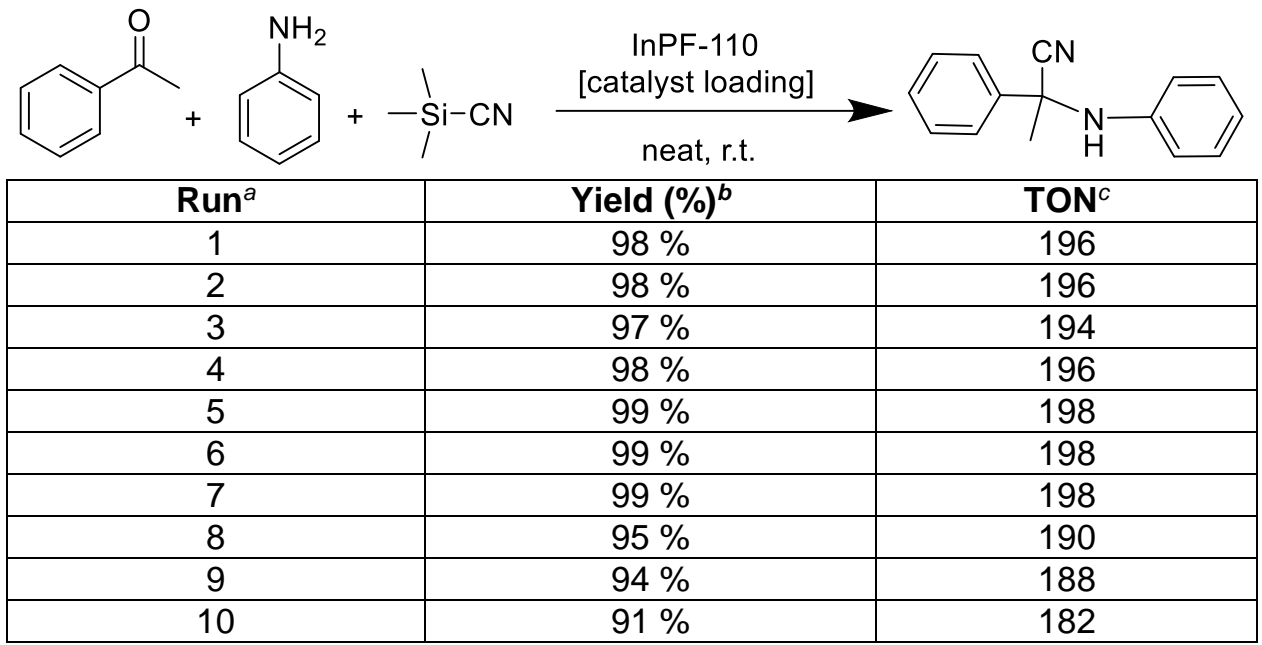

${ }^{a}$ Reaction conditions: $\mathrm{N}_{2}$ atmosphere, without solvent, at room temperature $\left(25^{\circ} \mathrm{C}\right)$, with a catalyst loading of $0.5 \mathrm{~mol} \%$. ${ }^{b}$ Yield calculated by ${ }^{1} \mathrm{H}$ NMR from reaction crude. ${ }^{c} \mathrm{TON}=$ (mol substrate $/ \mathrm{mol}$ catalyst) .

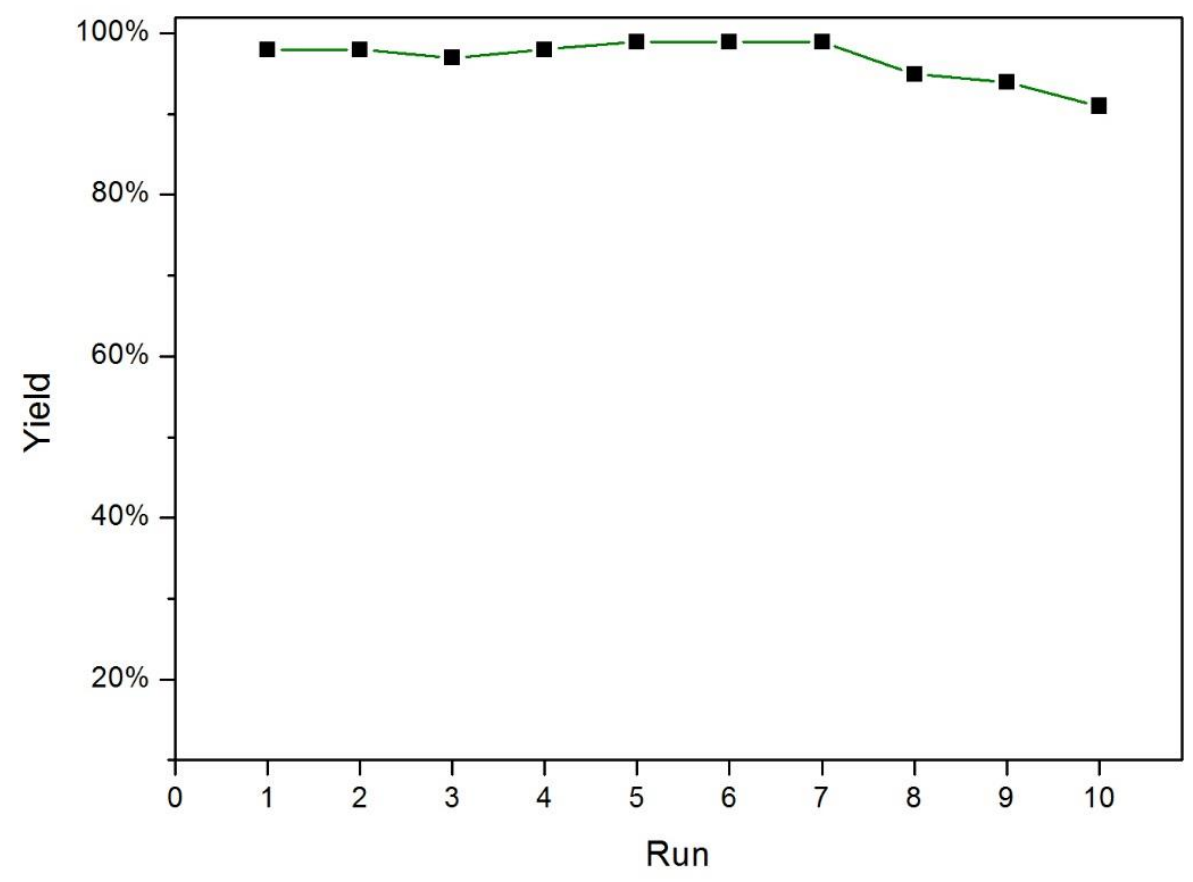

Figure S7.1 Graph with recycle run yields obtained, from the first to the tenth. 


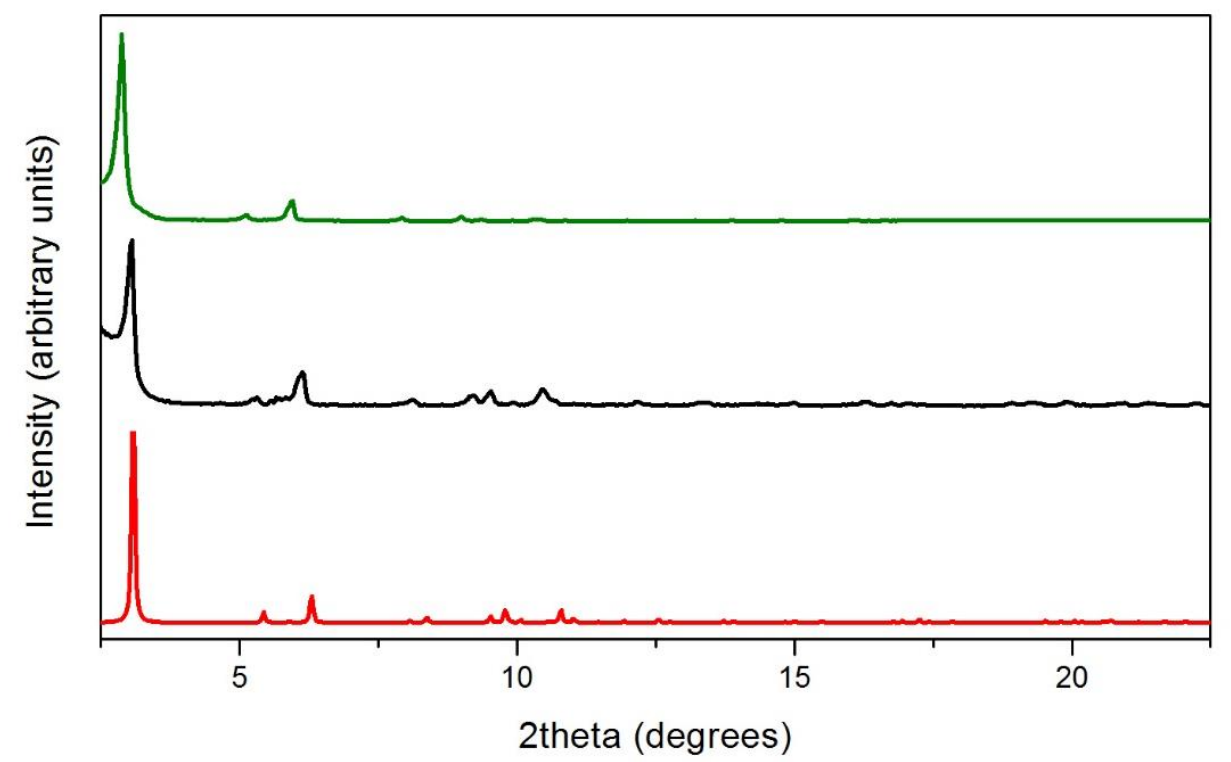

Figure S7.2 Simulated profile from single-crystal diffraction structure data (red), experimental profile from powder $\mathrm{X}$-ray diffraction (black) and after the tenth run in catalytic activity experiments (green). 
- S8. CHARACTERIZATION OF THE STRECKER 3C REACTION PRODUCT

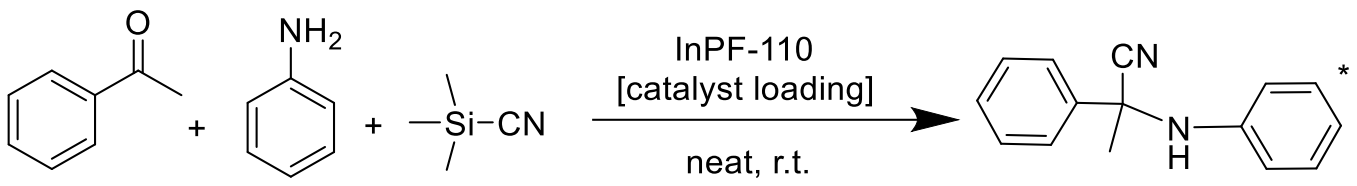

\section{1*2-Phenyl-2-(phenylamino)propanenitrile}

White solid: ${ }^{1} \mathrm{H}$ NMR (300 MHz, $\left.\mathrm{CDCl}_{3}\right): \delta=1.96(\mathrm{~s}, 3 \mathrm{H}), 4.29$ (brs, $\left.1 \mathrm{H}, \mathrm{NH}\right), 6.54-6.57(\mathrm{~d}, 2 \mathrm{H})$, 6.79-6.84 (t, 1H), 7.10-7.15 (t, 2H), 7.38-7.42 (m, 3H), 7.62-7.66 (m, 2H). IR (KBr) v(cm $\left.{ }^{-1}\right): 3387$ $v(\mathrm{~N}-\mathrm{H}), 3053,2991,2221 v(\mathrm{C}-\mathrm{N}), 1600,1513,1482,1446,1374,1312,1262,1215,1169$, 1102, 1066, 913, 856, 743, 692, 625 (which corresponds with the spectrum reported for compound with CAS No. 17424-68-9). FAB: m/z: 222 [M+], 222, 196, 180. This compound is known. ${ }^{6}$

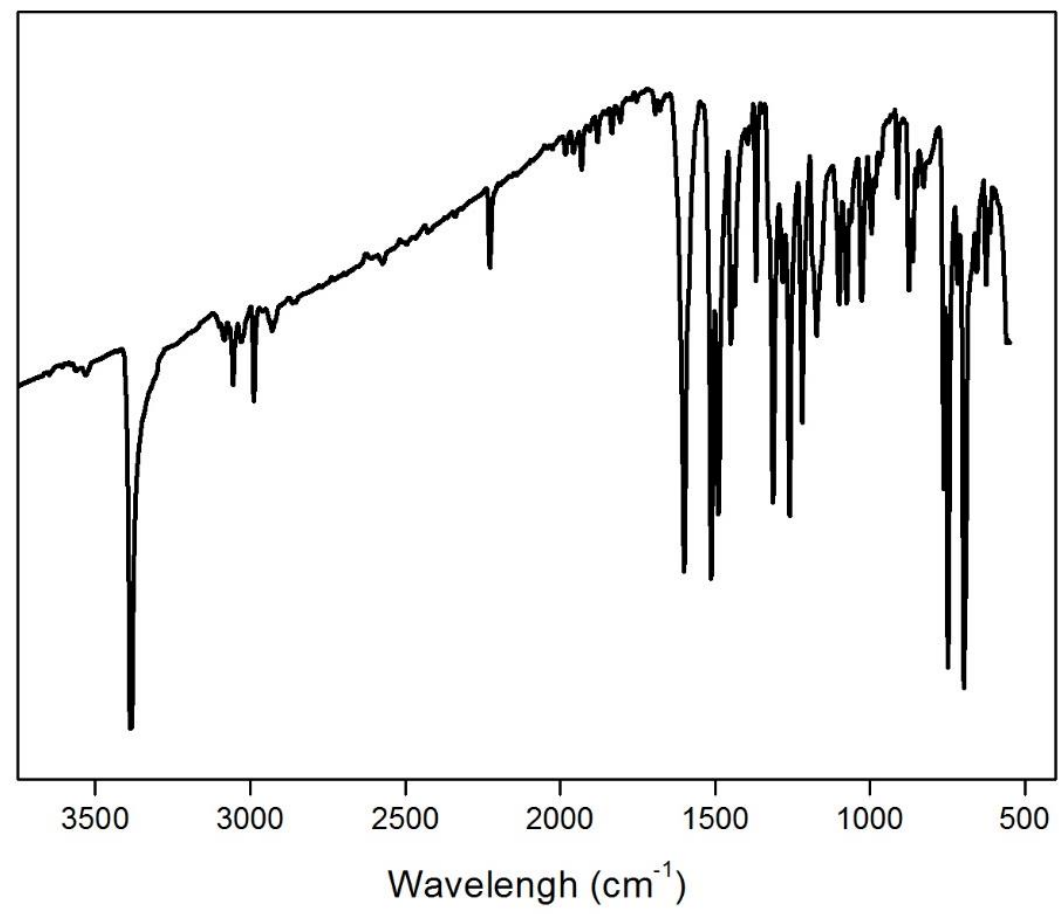

Figure S8.1a Infrared spectra of 2-Phenyl-2-(phenylamino)propanenitrile. 


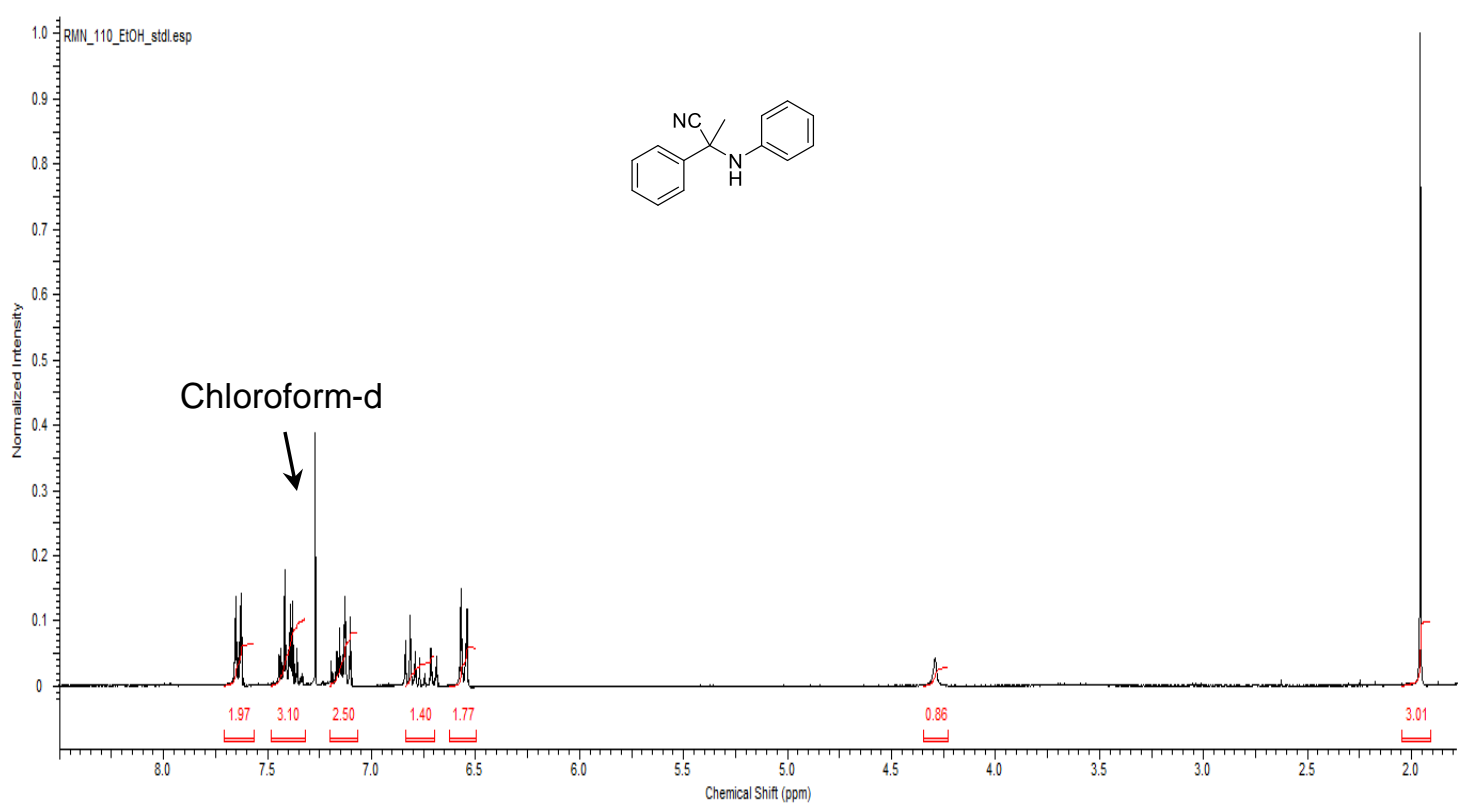

Figure S8.1b ${ }^{1} \mathrm{H}$ NMR spectra of 2-Phenyl-2-(phenylamino)propanenitrile. (Table 1, entry 2; table S6, entry 3).

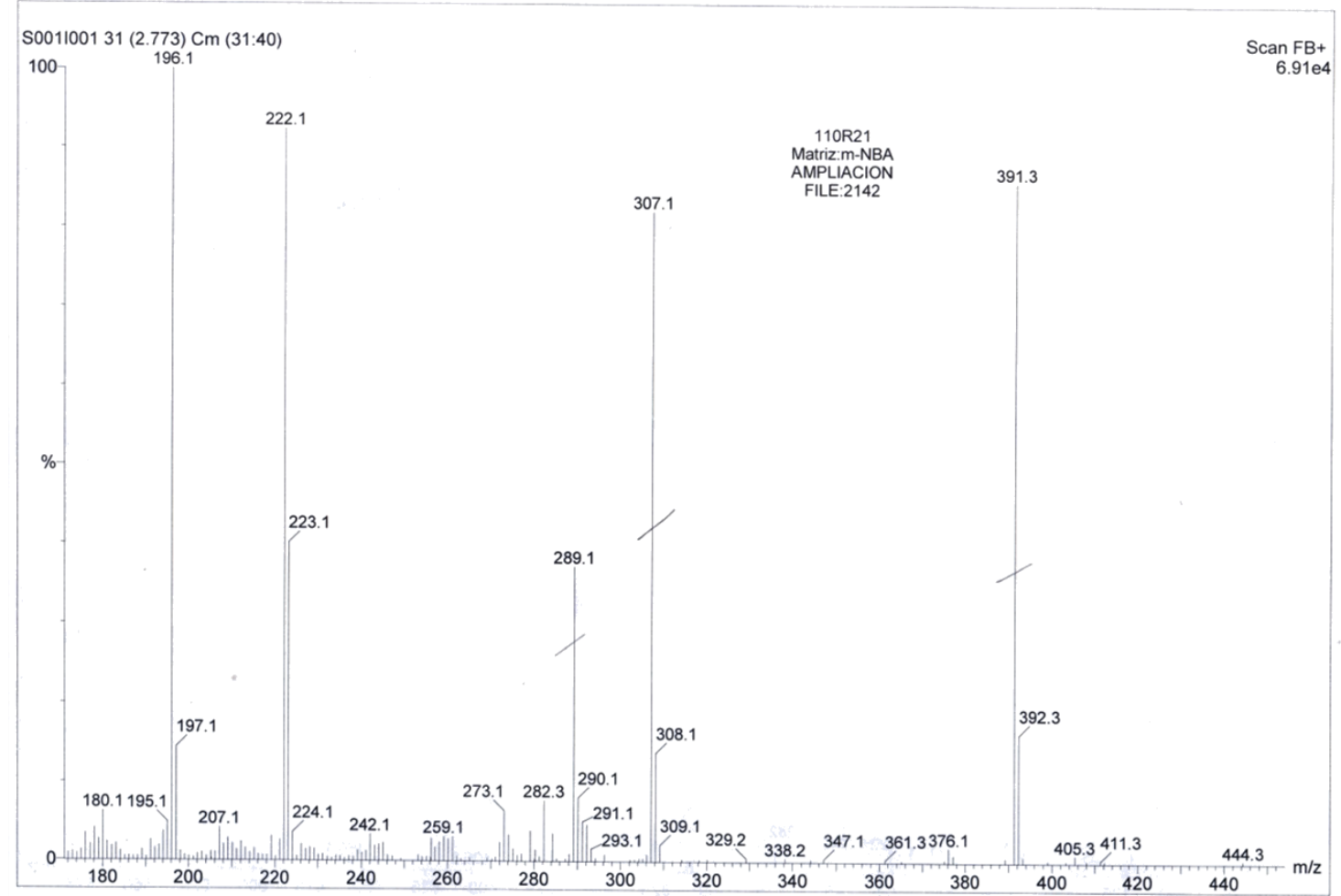

Figure S8.1c ESI-MS spectra of 2-Phenyl-2-(phenylamino)propanenitrile. Molecular weight: 222; without $\mathrm{CN}: 196$. Strikethrough signals correspond to the matrix. 


\subsection{Characterization of 3C Strecker Reaction ketone and amine scope}<smiles>Cc1ccc(C(C)(C#N)Nc2ccccc2)cc1</smiles>

Table 1, entries 4 and 5. Benzeneacetonitrile, $\alpha, 4$-dimethyl- $\alpha$-(phenylamino) ${ }^{6}$

CAS Registry Number 17424-69-0;

${ }^{1} \mathrm{H}$ NMR $\left(300 \mathrm{MHz} ; \mathrm{CDCl}_{3}\right): \delta=1.95(\mathrm{~s}, 3 \mathrm{H}), 2.38(\mathrm{~s}, 3 \mathrm{H}), 4.27$ (brs, $\left.1 \mathrm{H}, \mathrm{NH}\right), 6.56-6.59(\mathrm{~d}, 2 \mathrm{H})$, 6.80-6.85 (t, 1H), 7.11-7.17 (m, 2H), 7.21-7.24 (d, 2H), 7.51-7.54 (d, 2H)<smiles>CC(C#N)(Nc1ccccc1)c1ccc(Cl)cc1</smiles>

Table 1, entries 6 and 7. Benzeneacetonitrile, 4-chloro- $\alpha$-methyl- $\alpha$-(phenylamino) ${ }^{14}$ CAS Registry Number 17424-70-3;

${ }^{1} \mathrm{H}$ NMR $\left(300 \mathrm{MHz} ; \mathrm{CDCl}_{3}\right): \delta=1.94(\mathrm{~s}, 3 \mathrm{H}), 4.28(\mathrm{brs}, 1 \mathrm{H}, \mathrm{NH}), 6.52-6.55(\mathrm{~d}, 2 \mathrm{H}), 6.81-6.86(\mathrm{t}$, $1 \mathrm{H})$, 7.12-7.17 (m, 2H), 7.37-7.40 (d, 2H), 7.56-7.59 (d, 2H)<smiles>CC(C#N)(Cc1ccccc1)Nc1ccccc1</smiles>

Table 1, entry 8. Benzenepropanenitrile, $\alpha$-methyl- $\alpha$-(phenylamino $)^{9}$ CAS Registry Number 203123-40-4;

${ }^{1} \mathrm{H}$ NMR (300 MHz; $\mathrm{CDCl}_{3}$ ): $\square=1.66(\mathrm{~s}, 3 \mathrm{H}), 3.15-3.34$ (dd, $\left.2 \mathrm{H}\right), 3.70$ (brs, $\left.1 \mathrm{H}, \mathrm{NH}\right), 6.70-6.73$ $(\mathrm{d}, 2 \mathrm{H}), 6.76-6.81(\mathrm{t}, 1 \mathrm{H}), 7.25-7.42(\mathrm{~m}, 7 \mathrm{H})$<smiles>CCCCC(C)(C)Nc1ccccc1</smiles>

Table 1, entry 9 and 10. Hexanenitrile, 2-methyl-2-(phenylamino) ${ }^{14}$ CAS Registry Number 516448-17-2;

GC-MS; retention time: $13.1 \mathrm{~min}$ (method: from 80 to $220^{\circ} \mathrm{C}$, rate of $9 \stackrel{\circ}{\circ} / \mathrm{min}$ ). MS: 175, 160, 147, 133, 119, 105, 93, 78<smiles>CCCC(C)(CC)Nc1ccccc1</smiles>

Table 1, entry 11. Pentanenitrile, 2-ethyl-2-(phenylamino) CAS Registry Number 1701631-00-6;

GC-MS; retention time: $12.8 \mathrm{~min}$ (method: from 80 to $220{ }^{\circ} \mathrm{C}$, rate of $9{ }^{\circ} \mathrm{C} / \mathrm{min}$ ). MS: 175,160 , 147, 133, 118, 105, 94, 78; FAB: m/z: $202[\mathrm{M}+], 176,132,94$. 
<smiles>N#CC1(Nc2ccccc2)CCCC1</smiles>

Table 1, entry 12 and 13. Cyclopentanecarbonitrile, 1-(phenylamino $)^{6}$ CAS Registry Number 6636-92-6;

${ }^{1} \mathrm{H}$ NMR $\left(300 \mathrm{MHz} ; \mathrm{CDCl}_{3}\right): \delta=1.85-1.95(\mathrm{~m}, 4 \mathrm{H}), 2.11-2.21(\mathrm{~m}, 2 \mathrm{H}), 2.35-2.45(\mathrm{~m}, 2 \mathrm{H}), 3.86$ (brs, $1 \mathrm{H}, \mathrm{NH}), 6.82-6.91(\mathrm{~m}, 3 \mathrm{H})$, 7.23-7.29 (m, 2H).<smiles>N#CC1(Nc2ccccc2)CCCCC1</smiles>

Table 1, entry 14. Cyclohexanecarbonitrile, 1-(phenylamino $)^{15}$ CAS Registry Number 64269-06-3;

${ }^{1} \mathrm{H}$ NMR $\left(300 \mathrm{MHz} ; \mathrm{CDCl}_{3}\right): \delta=, 1.62-1.83(\mathrm{~m}, 6 \mathrm{H}), 2.32-2.40(\mathrm{~m}, 4 \mathrm{H}), 3.26$ (brs, $\left.1 \mathrm{H}, \mathrm{NH}\right), 6.90-$ $6.96(\mathrm{~m}, 3 \mathrm{H}), 7.23-7.28(\mathrm{~m}, 2 \mathrm{H})$<smiles>CC(C#N)(Nc1ccccc1Br)c1ccccc1</smiles>

Table 2, entry 15. Benzeneacetonitrile, $\alpha$-[(2-bromophenyl)amino]-a-methyl-;

${ }^{1} \mathrm{H}$ NMR $\left(300 \mathrm{MHz} ; \mathrm{CDCl}_{3}\right): \delta=2.04(\mathrm{~s}, 3 \mathrm{H}), 3.65$ (brs, $\left.1 \mathrm{H}, \mathrm{NH}\right), 6.39-6.42(\mathrm{~d}, 1 \mathrm{H}), 6.61-6.70$ $(\mathrm{m}, 1 \mathrm{H}), 6.97-7.02(\mathrm{t}, 1 \mathrm{H}), 7.34-7.50(\mathrm{~m}, 5 \mathrm{H}), 7.59-7.62(\mathrm{~d}, 1 \mathrm{H}) .{ }^{13} \mathrm{C} \mathrm{NMR}\left(300 \mathrm{MHz} ; \mathrm{CDCl}_{3}\right): \delta=$ 33.23 (s, 1C), 56.74 (s, 1C, C cuaternary), 110.91 (s, 1C), 115.07 (s, 1C), 120.32 (s, 1C, CN), 124.62-129.95 (ss, Caromatics), 132.45 (s, 1C), 139.16 (s, 1C). FAB: m/z: 301 [M+], 274, 221, 171, $154,130$.<smiles>COc1ccc(NC(C)(C#N)c2ccccc2)cc1</smiles>

Table 2, entry 16. Benzeneacetonitrile, $\alpha$-[(4-methoxyphenyl)amino]- $\alpha$-methyl ${ }^{6}$ CAS Registry Number 81512-12-1;

${ }^{1} \mathrm{H}$ NMR $\left(300 \mathrm{MHz} ; \mathrm{CDCl}_{3}\right): \delta=2.02(\mathrm{~s}, 3 \mathrm{H}), 3.71(\mathrm{~s}, 3 \mathrm{H}), 3.80(\mathrm{brs}, 1 \mathrm{H}, \mathrm{NH}), 6.64-6.72(\mathrm{~m}, 4 \mathrm{H})$, 7.38-7.42 (m, 3H), 7.64-7.67 (m, 2H) 


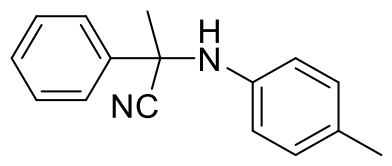

Table 2, entry 17 and 18. Benzeneacetonitrile, $\alpha$-methyl- $\alpha-[(4-m e t h y \mid p h e n y l) a m i n o]^{6}$ CAS Registry Number 81512-08-5;

${ }^{1} \mathrm{H}$ NMR $\left(300 \mathrm{MHz} ; \mathrm{CDCl}_{3}\right): \delta=1.94(\mathrm{~s}, 3 \mathrm{H}), 2.21(\mathrm{~s}, 3 \mathrm{H}), 4.15$ (brs, $\left.1 \mathrm{H}, \mathrm{NH}\right), 6.46-6.49(\mathrm{~d}, 2 \mathrm{H})$, 6.92-6.95 (d, 2H), 7.35-7.44 (m, 3H), 7.62-7.66 (m, 2H)

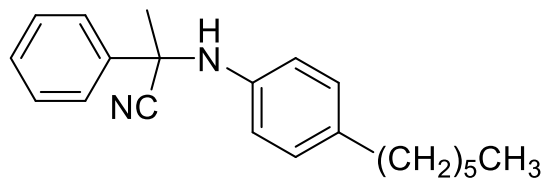

Table 2, entry 19 and 20. Benzeneacetonitrile, $\alpha$-methyl- $\alpha-[(4-$ hexylphenyl)amino]

${ }^{1} \mathrm{H}$ NMR $\left(300 \mathrm{MHz} ; \mathrm{CDCl}_{3}\right): \delta=0.85-0.89(\mathrm{~m}, 3 \mathrm{H}), 1.27-1.34(\mathrm{~m}, 6 \mathrm{H}), 1.47-1.52(\mathrm{t}, 2 \mathrm{H}), 1.94(\mathrm{~s}$, $3 \mathrm{H}), 2.43-2.48(\mathrm{t}, 2 \mathrm{H}), 4.15(\mathrm{brs}, 1 \mathrm{H}, \mathrm{NH}), 6.47-6.50(\mathrm{~m}, 2 \mathrm{H}), 6.92-6.95(\mathrm{~m}, 2 \mathrm{H}), 7.35-7.44(\mathrm{~m}$, $3 \mathrm{H}), 7.63-7.66(\mathrm{~m}, 2 \mathrm{H}) .{ }^{13} \mathrm{C} \mathrm{NMR}\left(300 \mathrm{MHz} ; \mathrm{CDCl}_{3}\right): \delta=14.15(\mathrm{~s}, 1 \mathrm{C}), 22.65(\mathrm{~s}, 1 \mathrm{C}), 29.05(\mathrm{~s}$, 1C), 31.55 (s, 1C), 31.78 (s, 1C), 33.27 (s, 1C), $35.10(\mathrm{~s}, 1 \mathrm{C}), 57.46$ (s, 1C, C cuaternary), 116.06 (s, 1C), 124.99 (s, 1C, CN), 128.54 (s, 2C), 128.89 (s, 3C), 129.21 (s, 2C), 134.56 (s, 1C), 140.25 (s, 1C), 141.41 (s, 1C).FAB: m/z: 306 [M+], 280, 264, 235, 222, 208, 196, 165, 177, 130, 106, 91, 77.

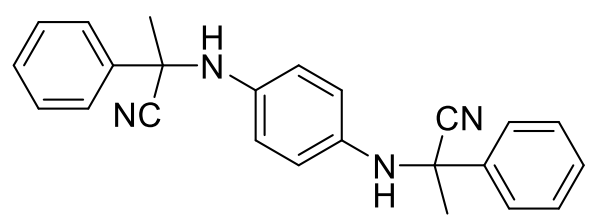

Table 2, entry 21 and 22. Benzeneacetonitrile, $\alpha, \alpha^{\prime}-\left(1,4-\right.$ phenylenediimino)bis[ $\alpha$-methyl-(9Cl)-; ${ }^{6}$ CAS Registry Number 866491-00-1;

${ }^{1} \mathrm{H}$ NMR $\left(300 \mathrm{MHz} ; \mathrm{CDCl}_{3}\right): \delta=1.88(\mathrm{~s}, 6 \mathrm{H}), 3.93(\mathrm{brs}, 2 \mathrm{H}, \mathrm{NH}), 6.40-6.41(\mathrm{~d}, 4 \mathrm{H}), 7.36-7.41$ $(\mathrm{m}, 6 \mathrm{H}), 7.59-7.63(\mathrm{~m}, 4 \mathrm{H})$ 
- S9. SPECTRAS FOR CHARACTERIZED COMPOUNDS

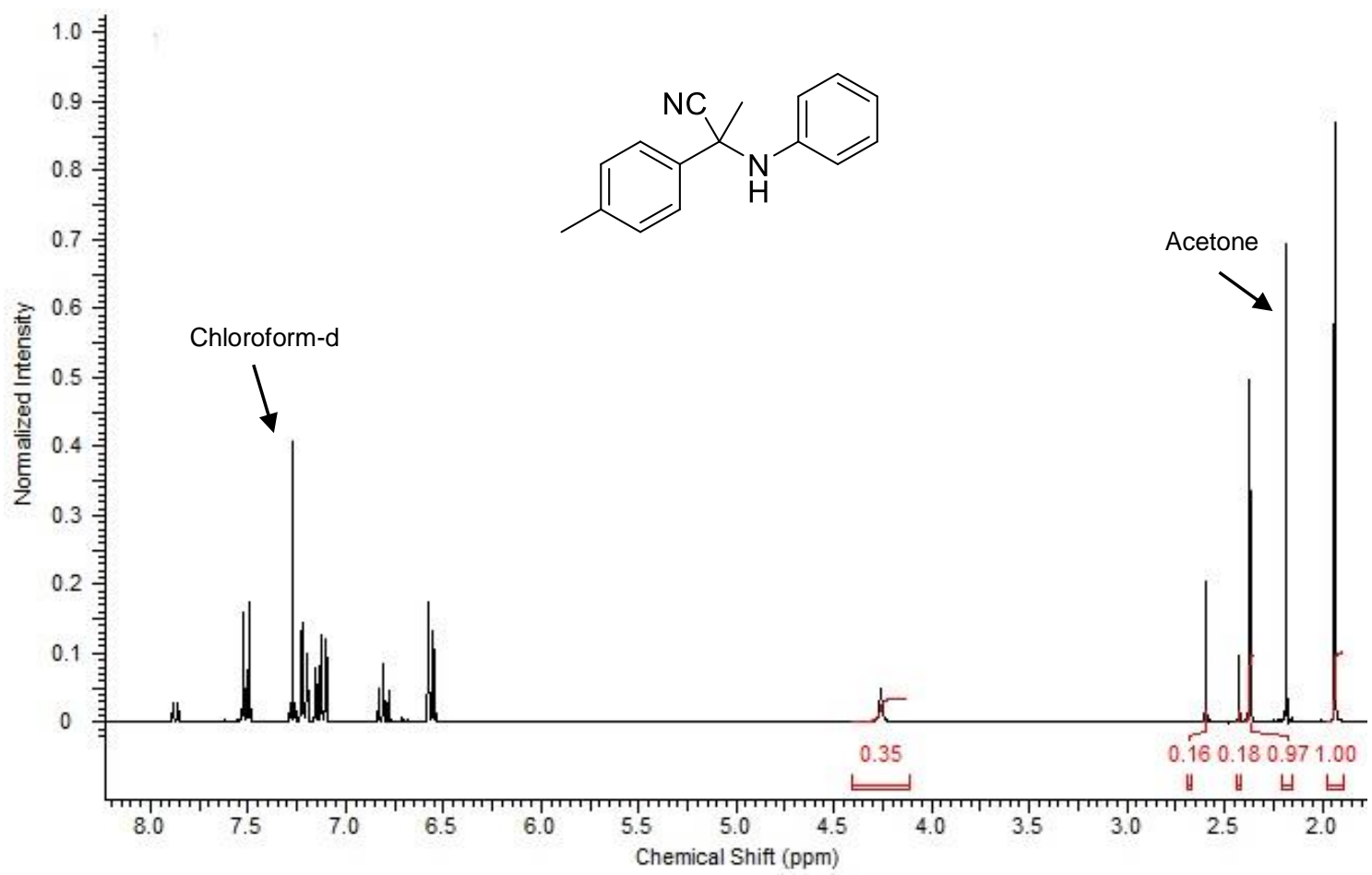

Figure S9.1 ${ }^{1} \mathrm{H}$ NMR spectra of Benzeneacetonitrile, $\alpha, 4$-dimethyl- $\alpha$-(phenylamino) ${ }^{6}$, (Table 1, entry 4).

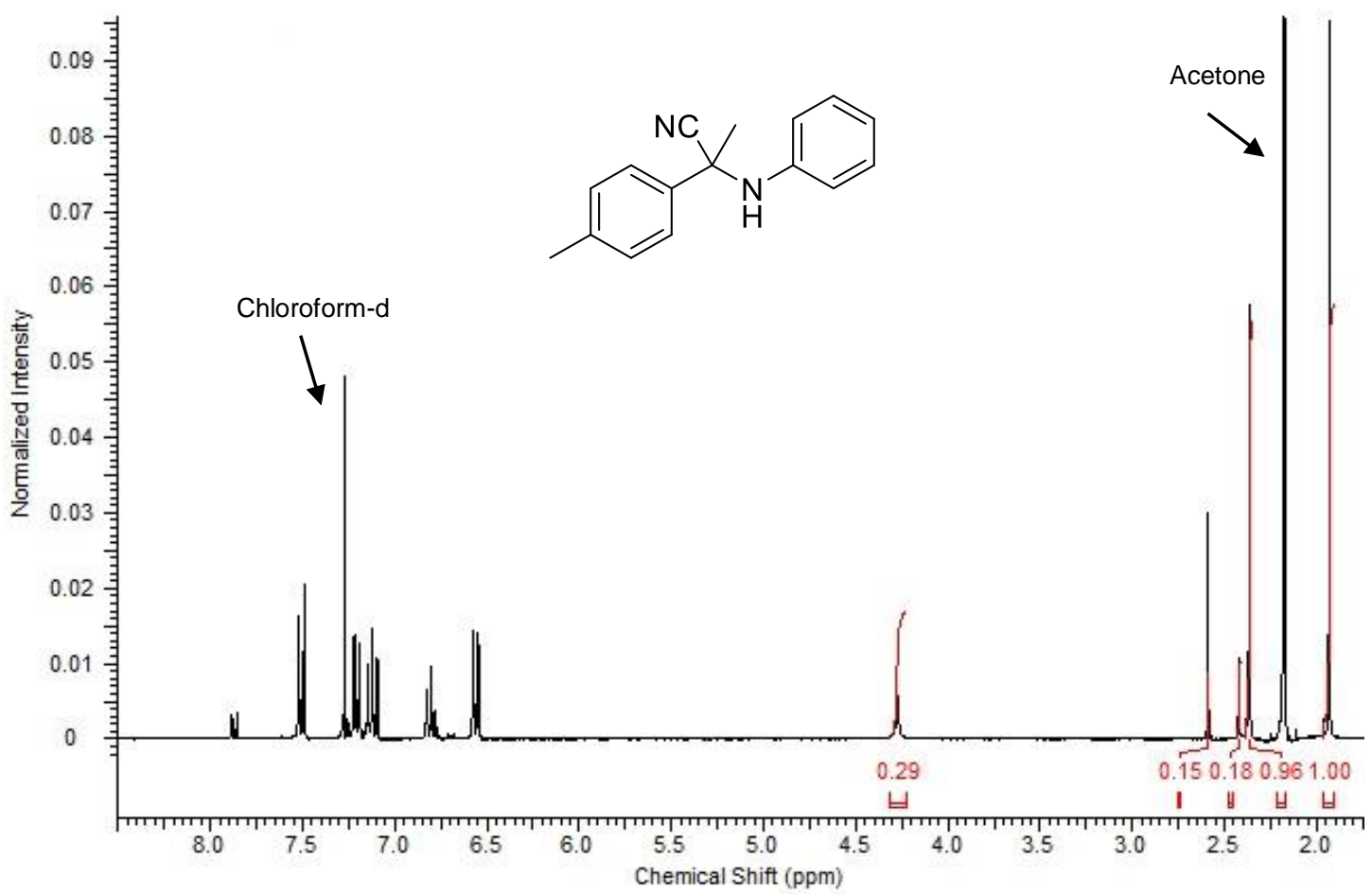

Figure S9.2 ${ }^{1} \mathrm{H}$ NMR spectra of Benzeneacetonitrile, $\alpha, 4$-dimethyl- $\alpha$-(phenylamino) ${ }^{6}$, (Table 1, entry 5). 


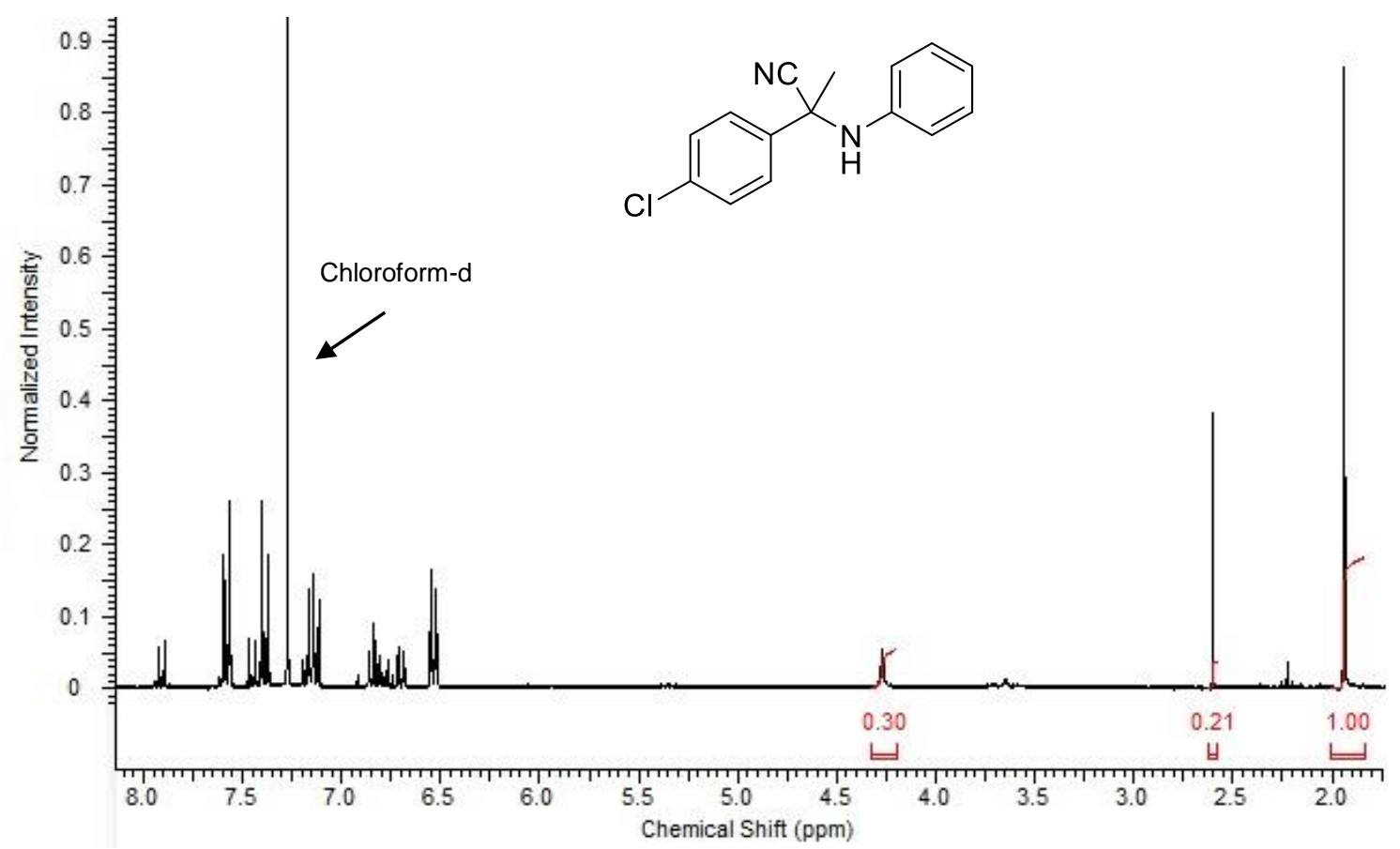

Figure S9.3 ${ }^{1} \mathrm{H}$ NMR spectra of Benzeneacetonitrile, 4-chloro- $\alpha$-methyl- $\alpha$-(phenylamino) ${ }^{14}$, (Table 1, entry 6).

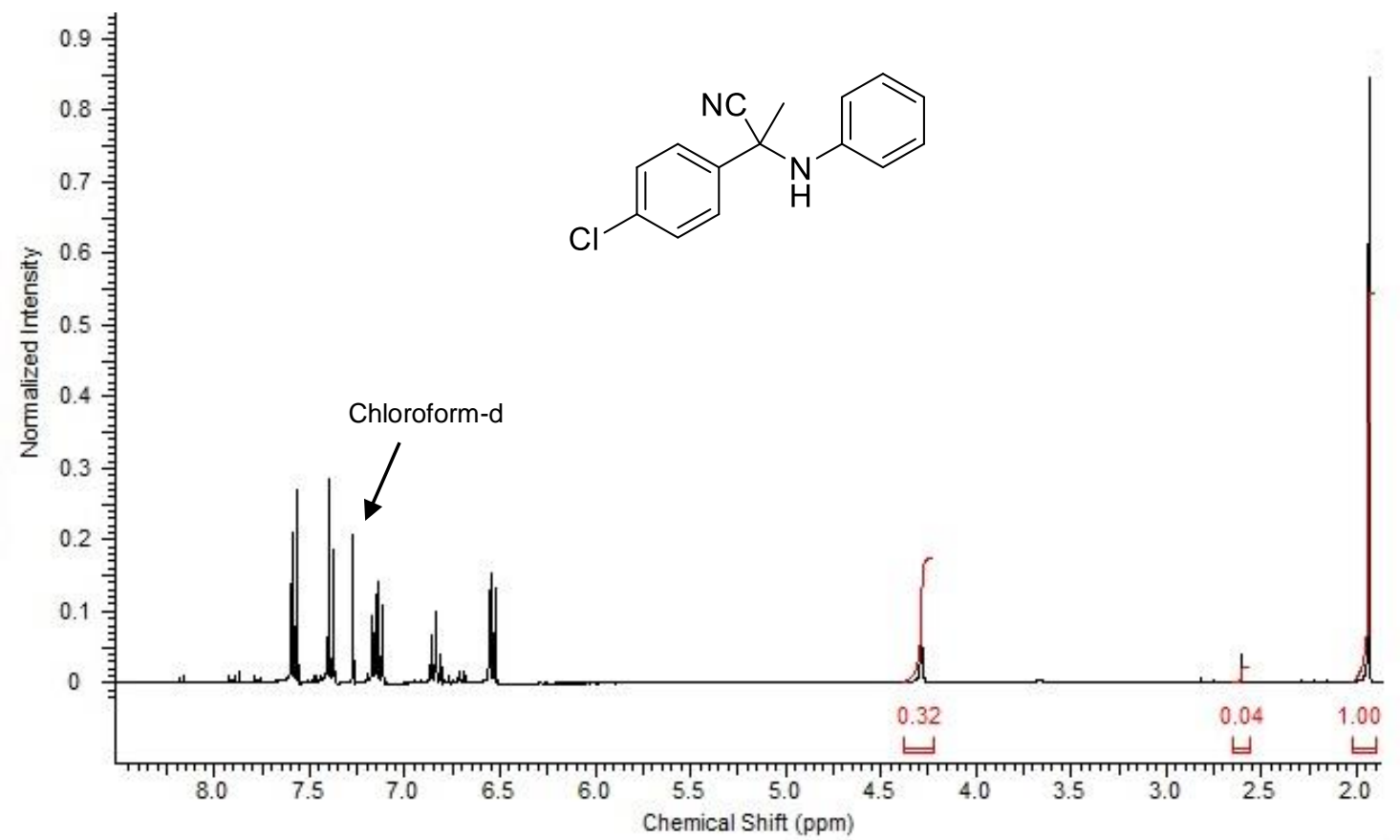

Figure S9.4 ${ }^{1} \mathrm{H}$ NMR spectra of Benzeneacetonitrile, 4-chloro- $\alpha$-methyl- $\alpha$-(phenylamino $)^{14}$, (Table 1, entry 7). 


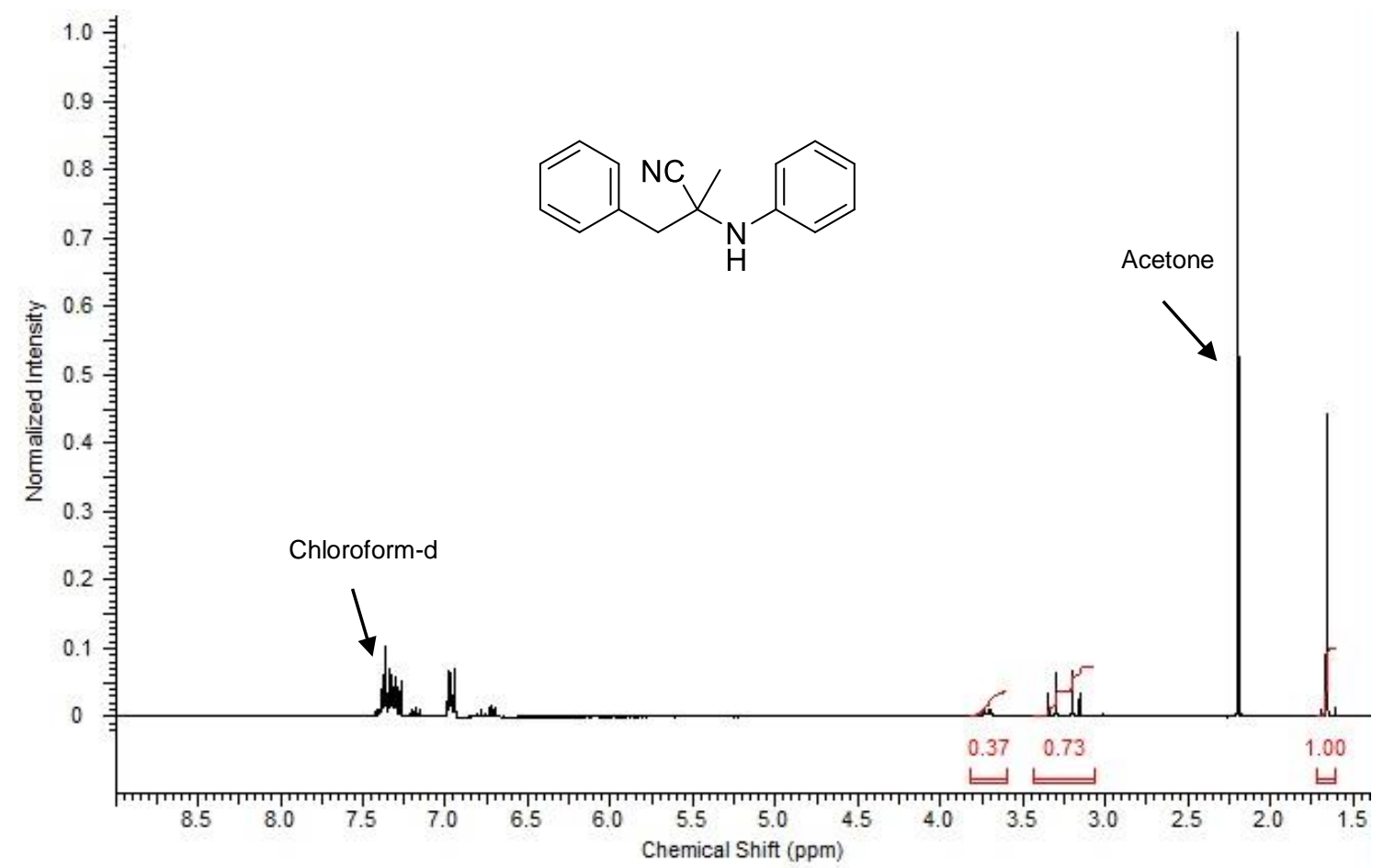

Figure S9.5 ${ }^{1} \mathrm{H}$ NMR spectra of Benzenepropanenitrile, $\alpha$-methyl- $\alpha$-(phenylamino) ${ }^{9}$, (Table 1, entry 8).

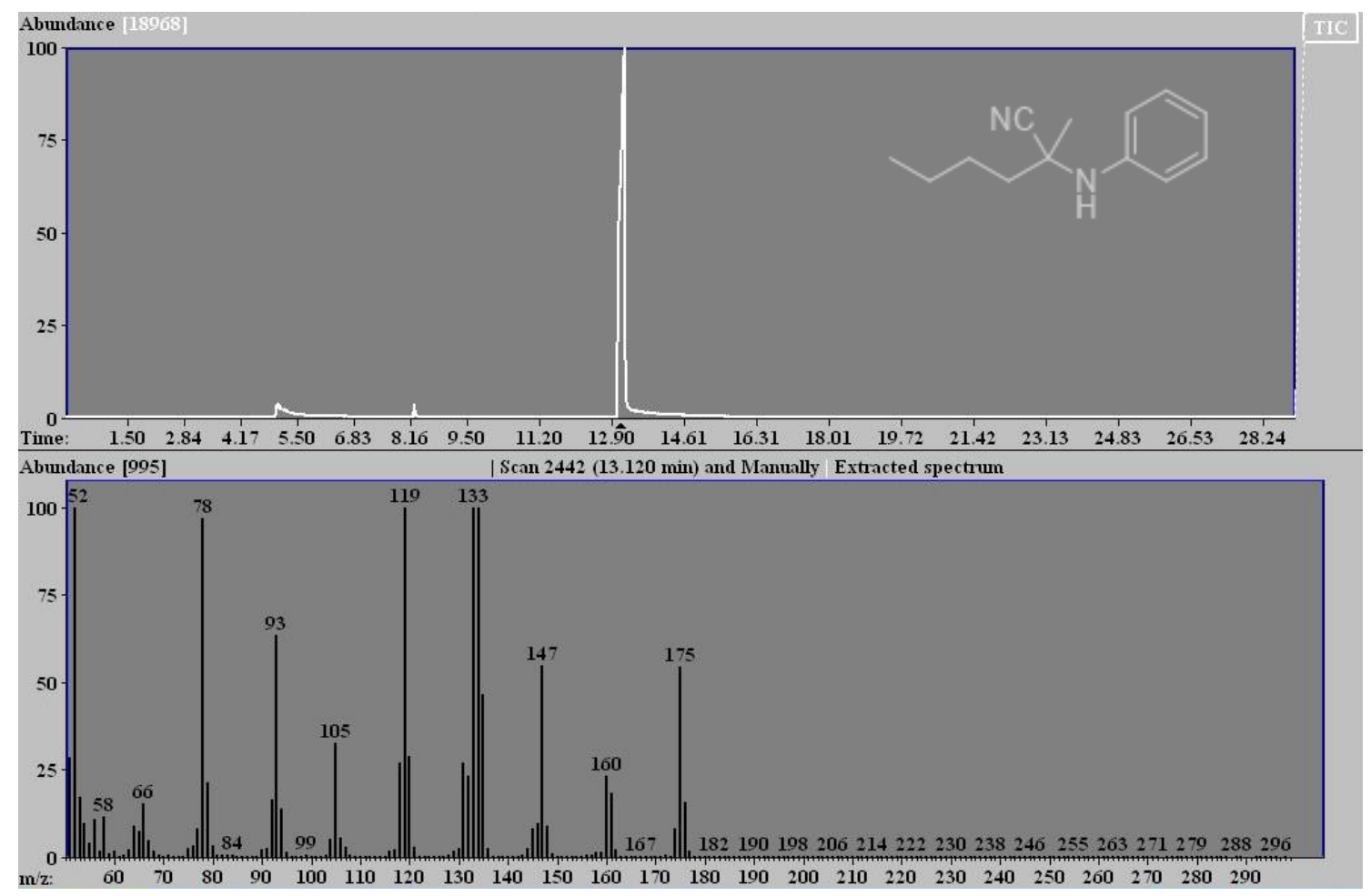

Figure S9.6 ${ }^{1} \mathrm{H}$ NMR spectra of Hexanenitrile, 2-methyl-2-(phenylamino $)^{14}$, (Table 1, entry 9). 


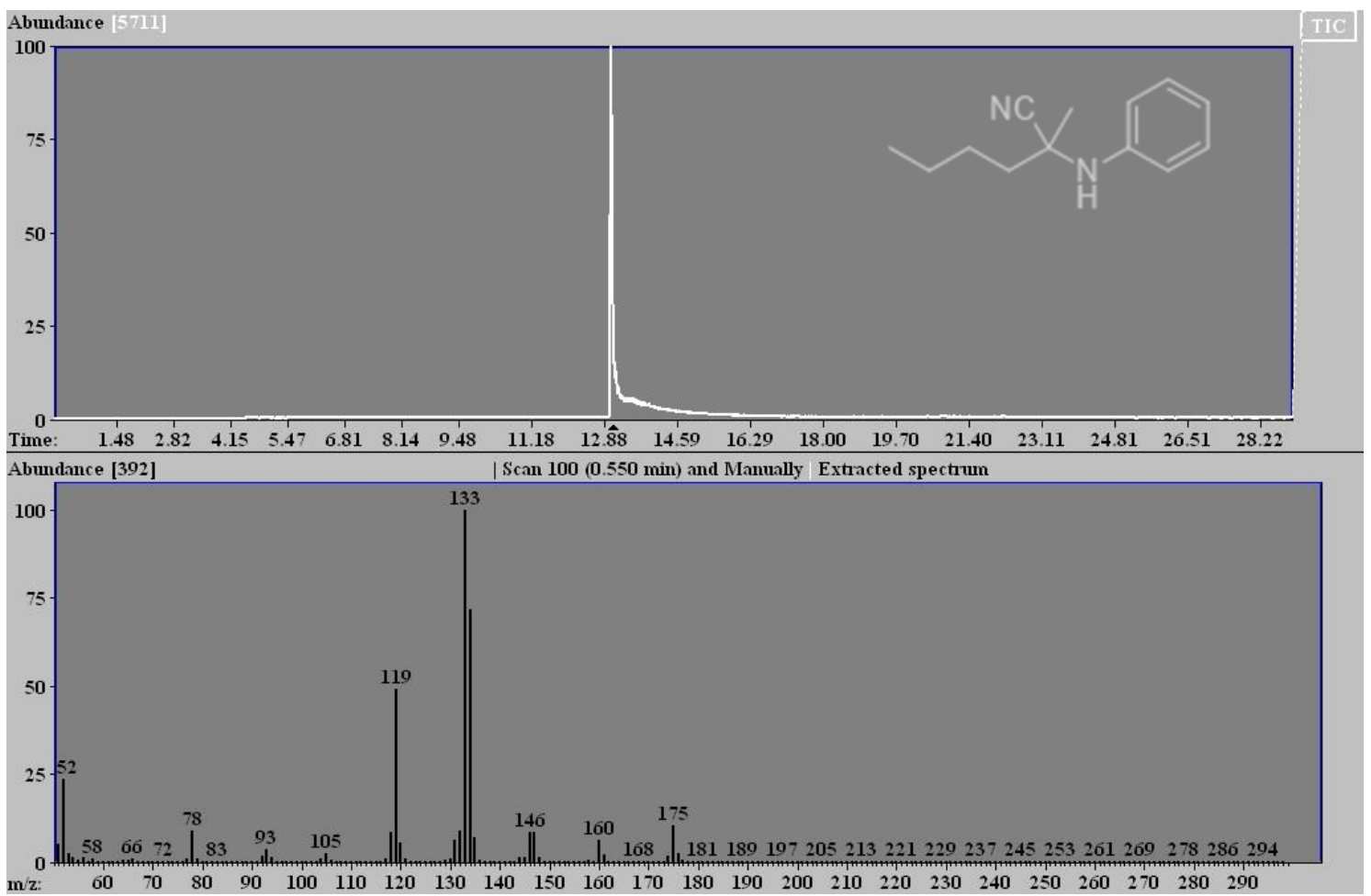

Figure S9.7 ${ }^{1} \mathrm{H}$ NMR spectra of Hexanenitrile, 2-methyl-2-(phenylamino) $)^{14}$, (Table 1, entry 10).

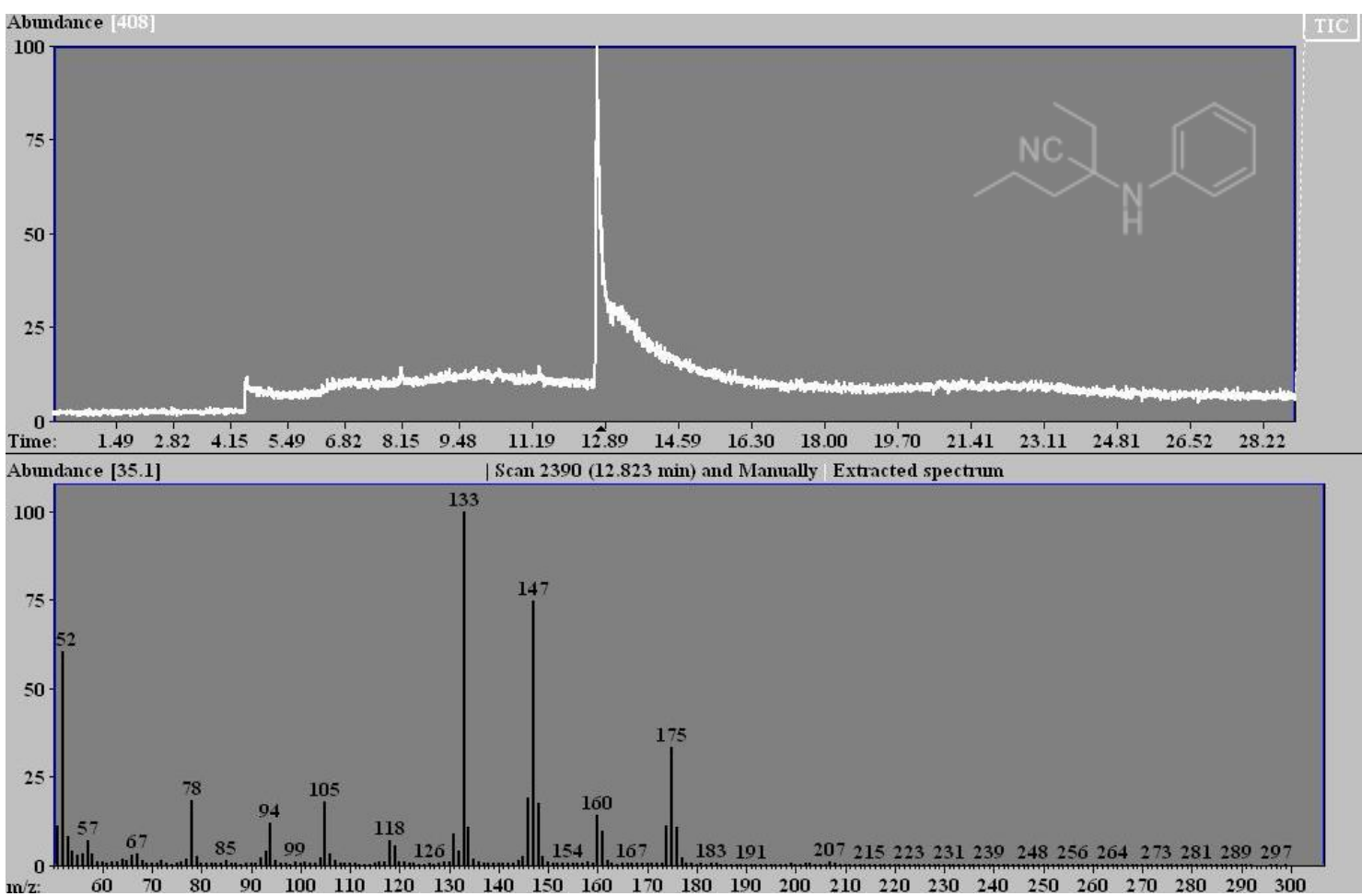

Figure S9.8 ${ }^{1} \mathrm{H}$ NMR spectra of Pentanenitrile, 2-ethyl-2-(phenylamino) ${ }^{6}$, (Table 1, entry 11). 


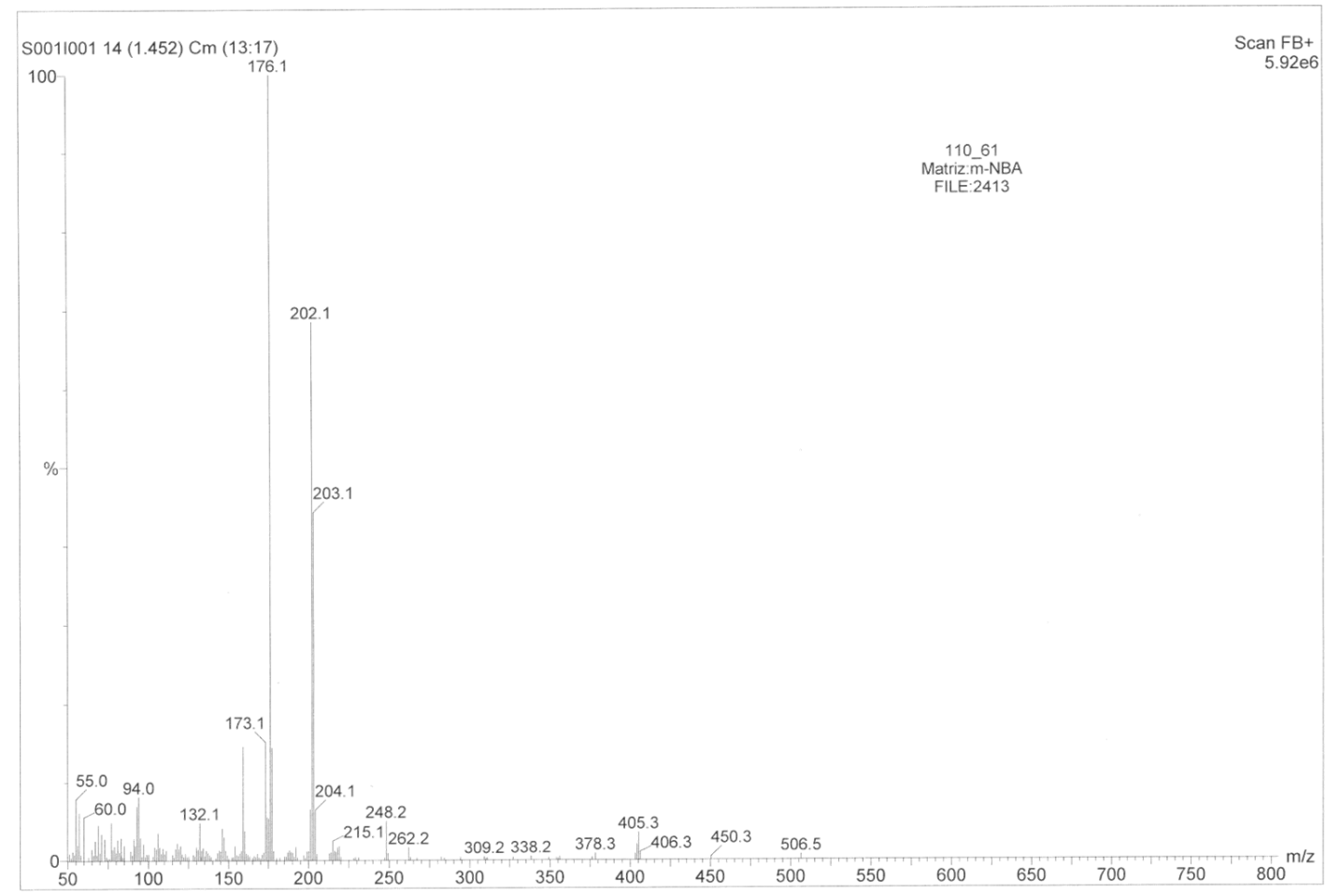

Figure S9.9 Mass spectra of Pentanenitrile, 2-ethyl-2-(phenylamino) ${ }^{6}$, (Table 1, entry 11).

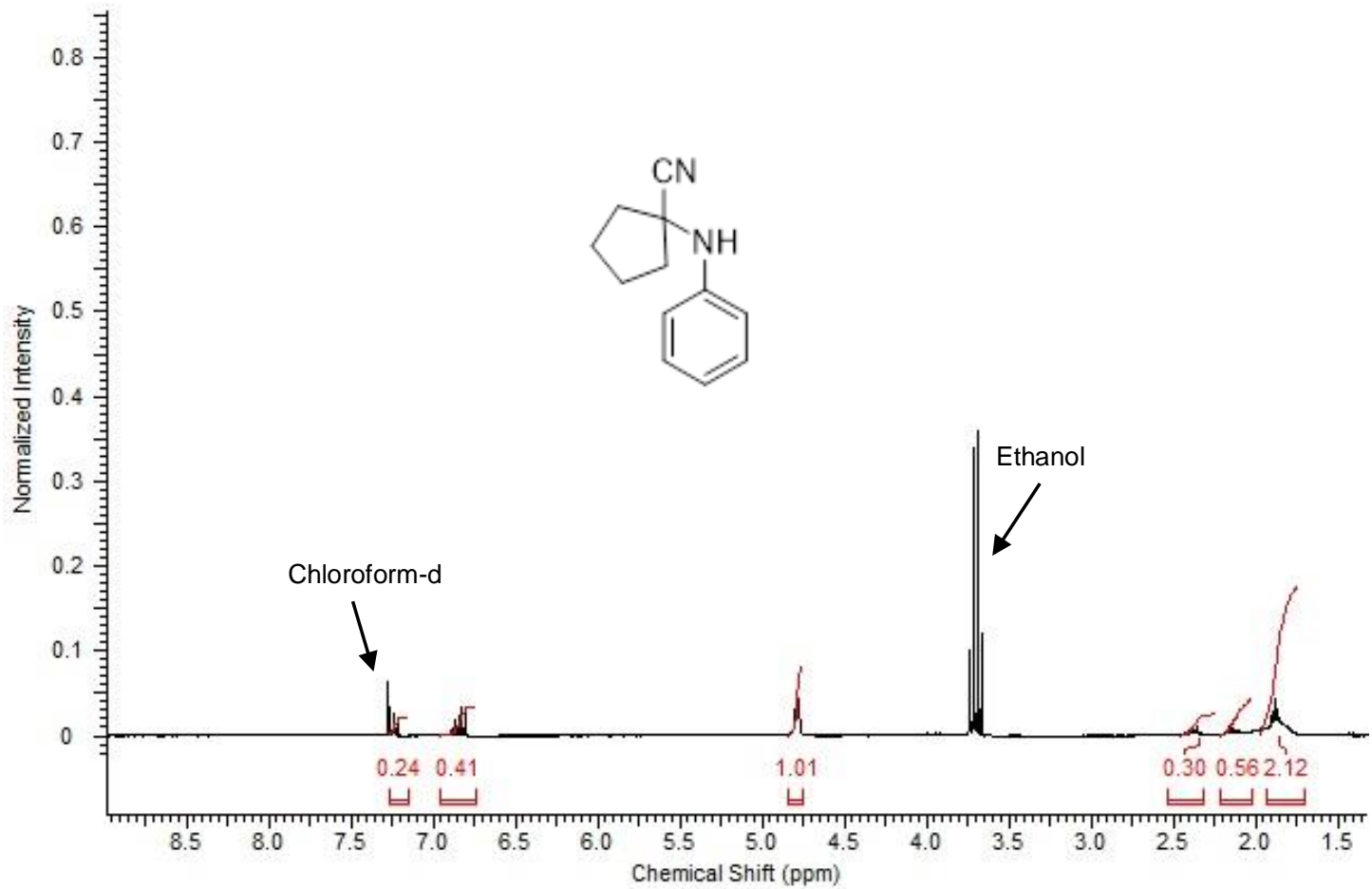

Figure S9.10 ${ }^{1} \mathrm{H}$ NMR spectra of Cyclopentanecarbonitrile, 1-(phenylamino) ${ }^{6}$, (Table 1, entry 12). 


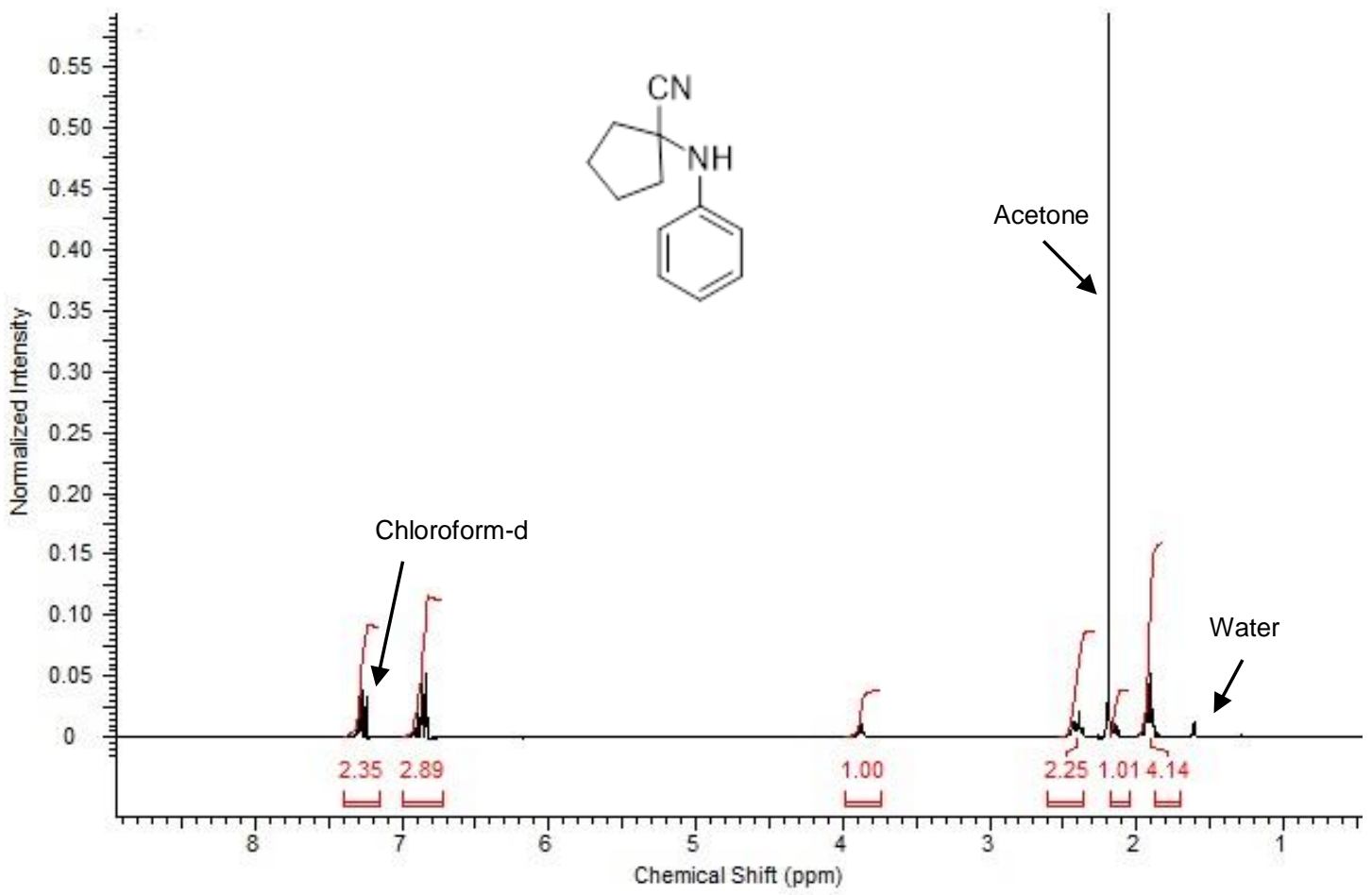

Figure S9.11 ${ }^{1} \mathrm{H}$ NMR spectra of Cyclopentanecarbonitrile, 1-(phenylamino) ${ }^{6}$, (Table 1, entry 13).

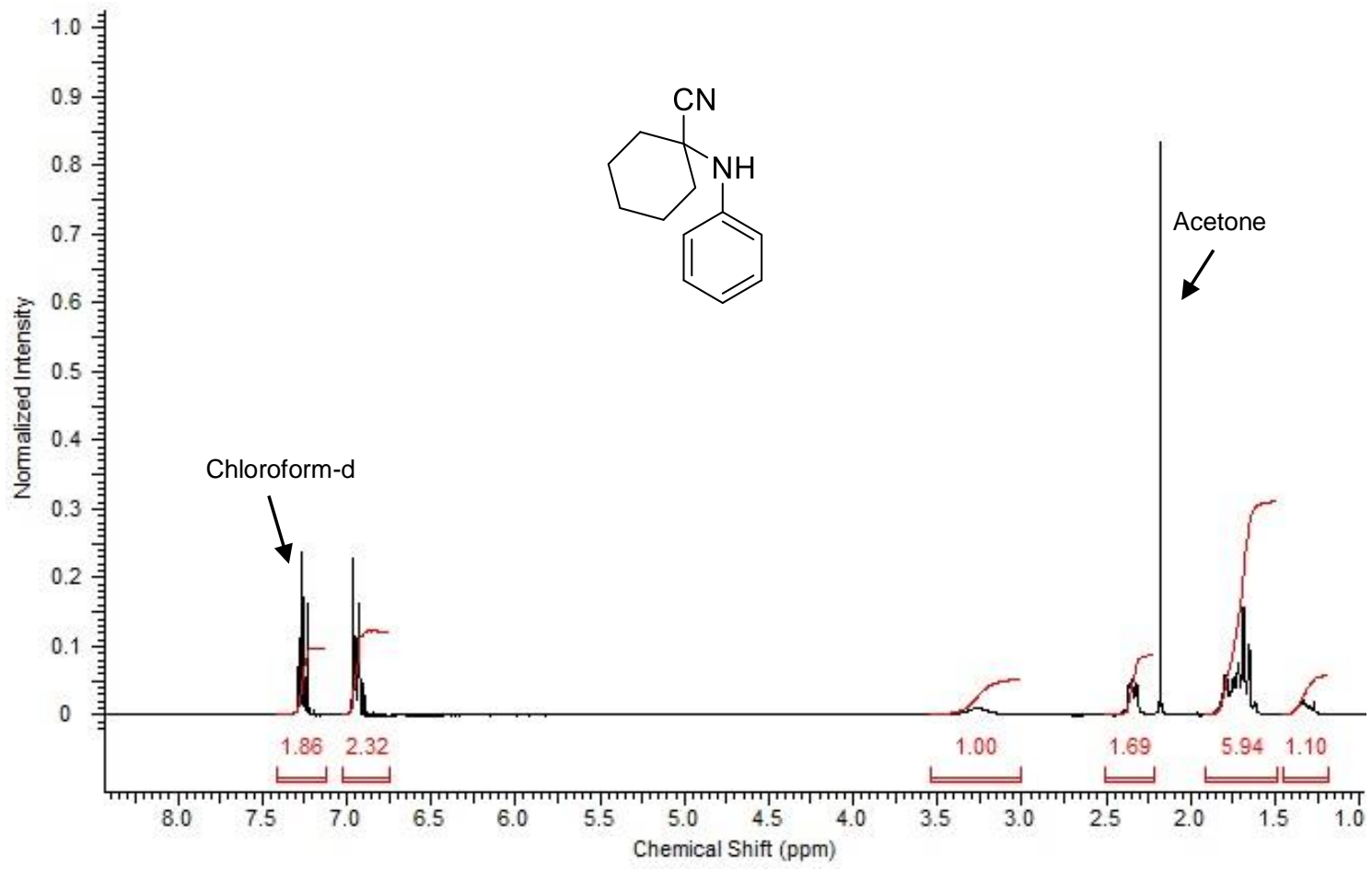

Figure S9.12 ${ }^{1} \mathrm{H}$ NMR spectra Cyclohexanecarbonitrile, 1 -(phenylamino $)^{15}$, (Table 1, entry 14). 


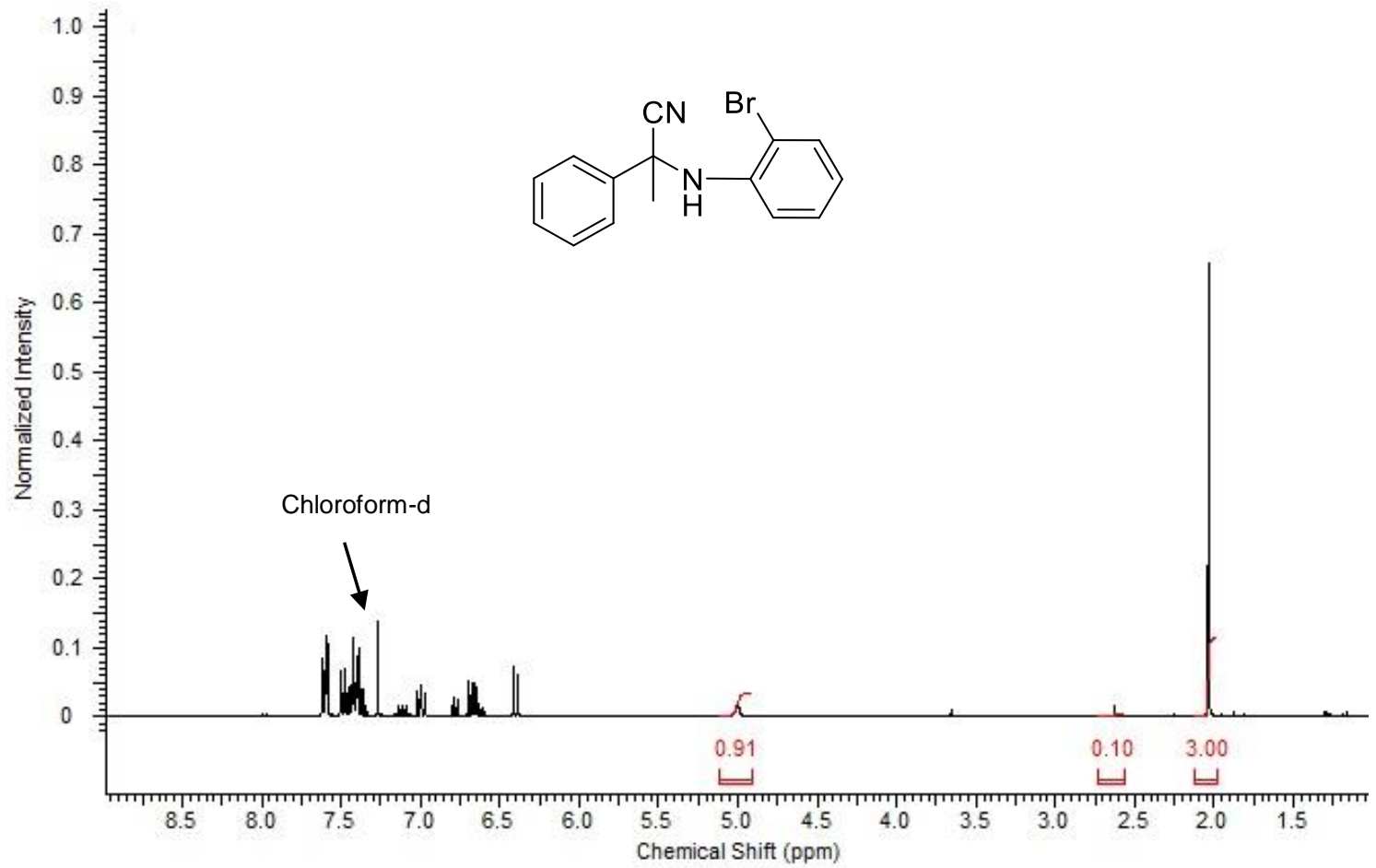

Figure S9.13 ${ }^{1} \mathrm{H}$ NMR spectra of Benzeneacetonitrile, $\alpha$-[(2-bromophenyl)amino]-a-methyl-, (Table 2, entry 15).

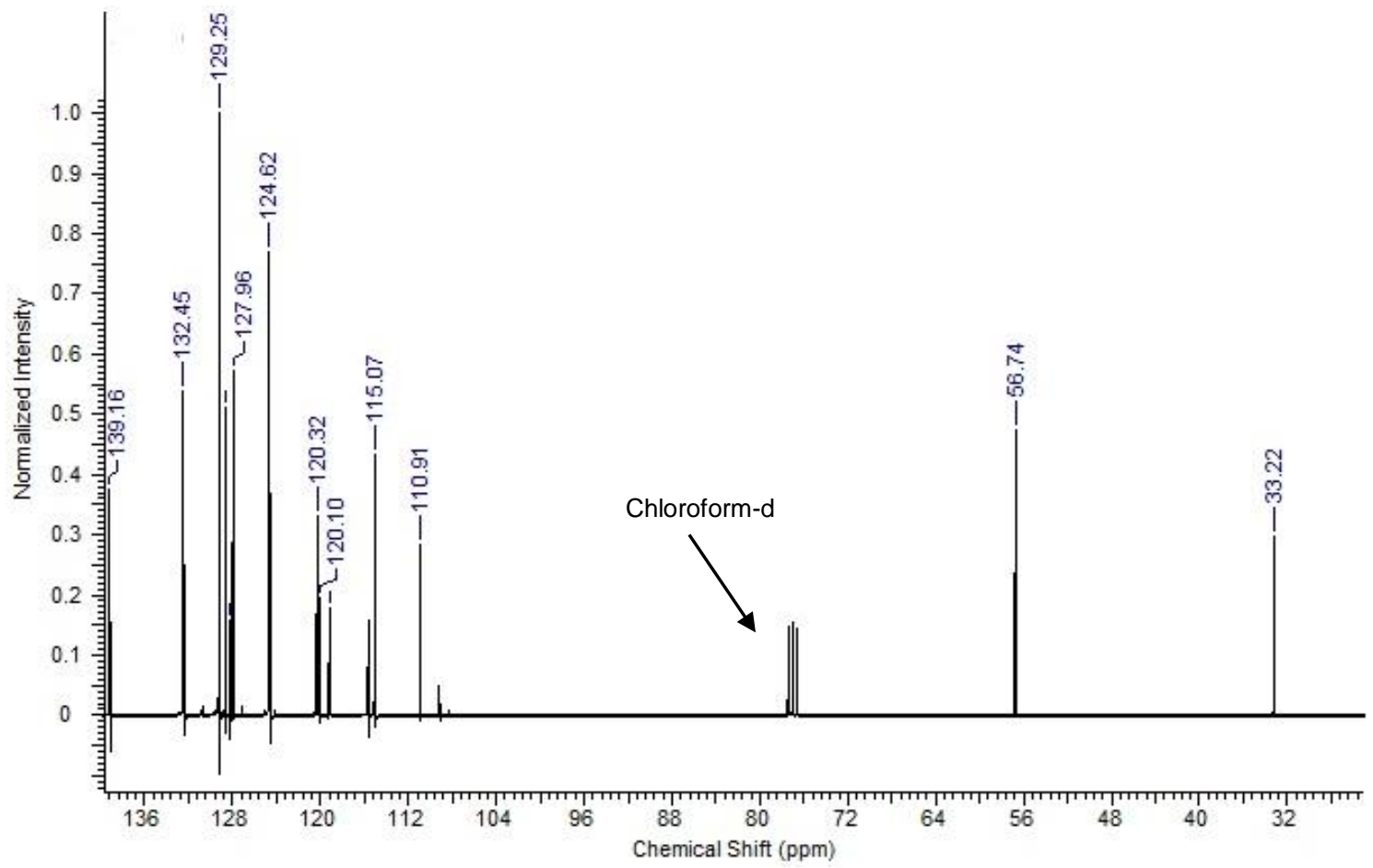

Figure S9.14 ${ }^{13} \mathrm{C}$ NMR spectra of Benzeneacetonitrile, $\alpha$-[(2-bromophenyl)amino]-a-methyl-, (Table 2, entry 15). 


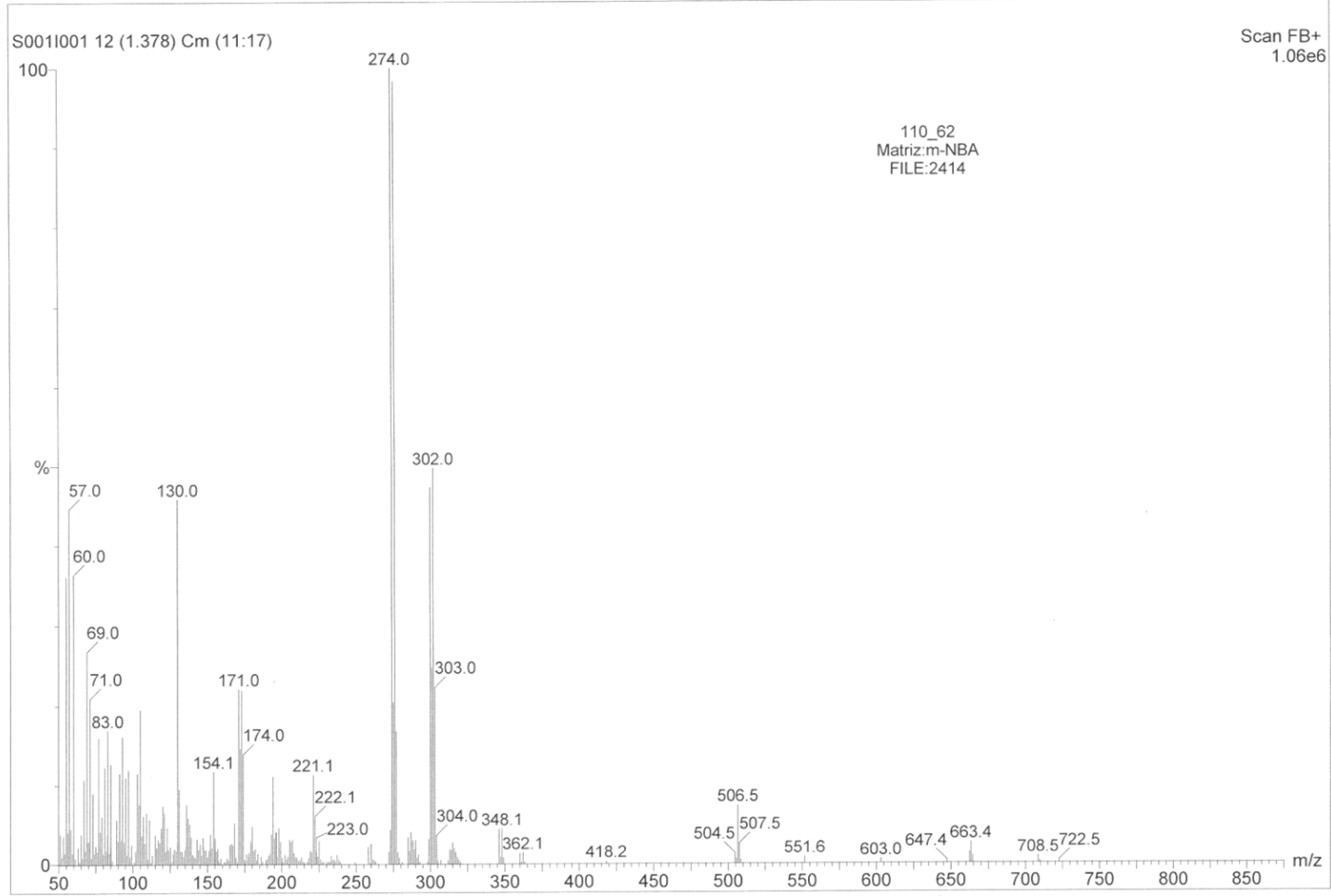

Figure S9.15 Mass spectra of Benzeneacetonitrile, $\alpha$-[(2-bromophenyl)amino]- $\alpha$-methyl-, (Table 2 , entry 15).

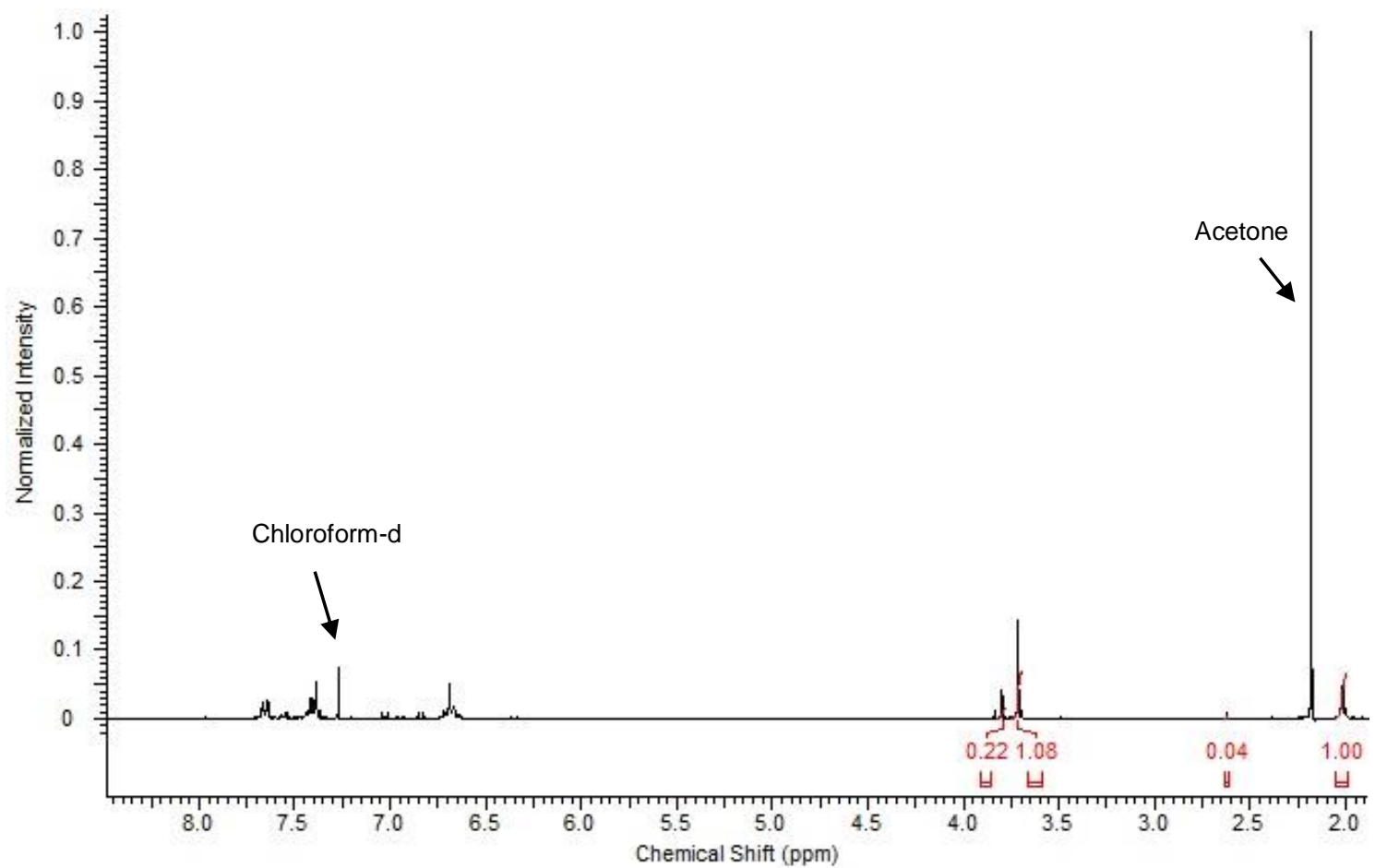

Figure S9.16 ${ }^{1} \mathrm{H}$ NMR spectra of Benzeneacetonitrile, $\alpha$-[(4-methoxyphenyl)amino]- $\alpha$-methyl ${ }^{6}$, (Table 2, entry 16). 


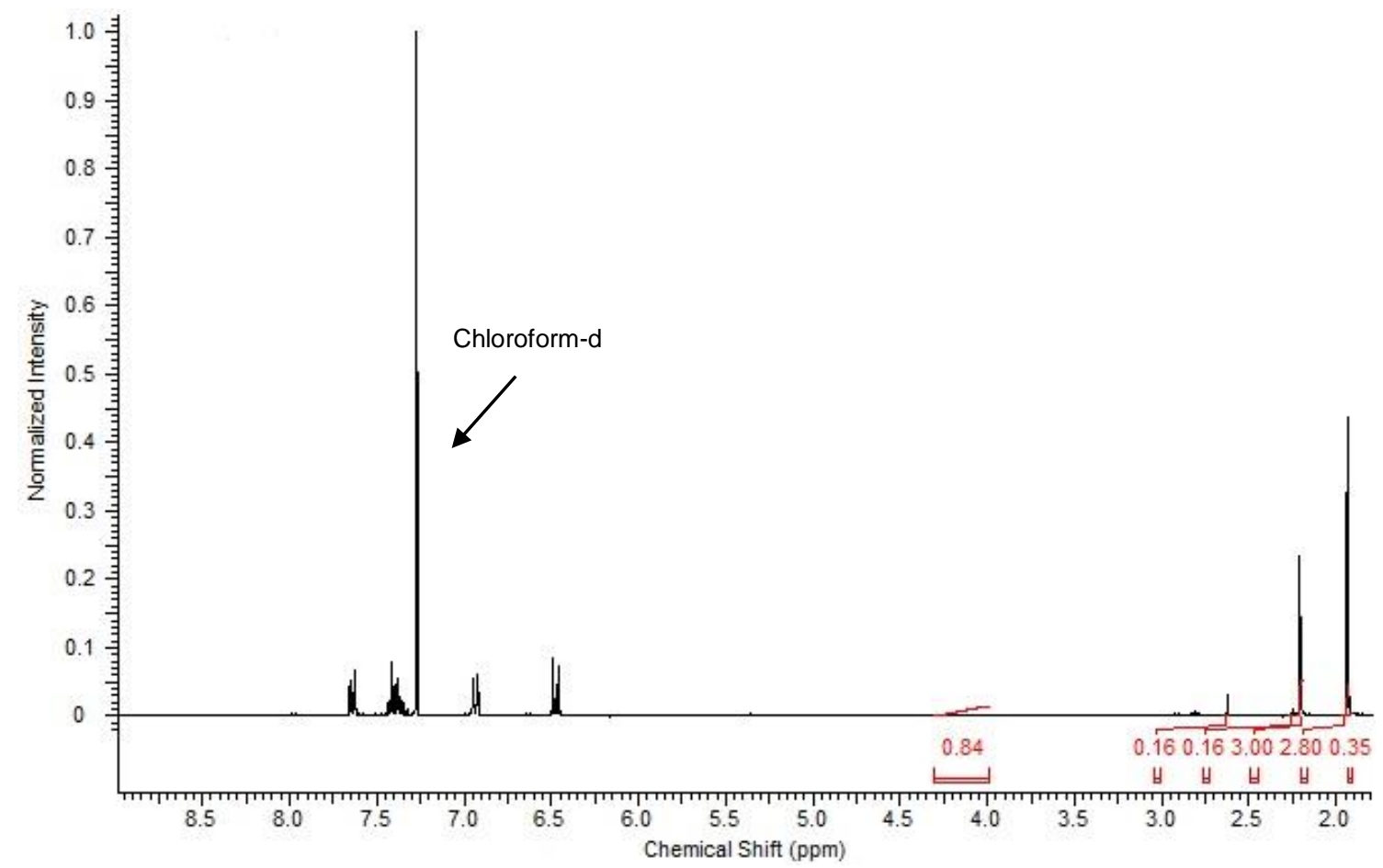

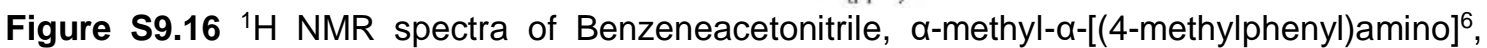
(Table 2, entry 17).

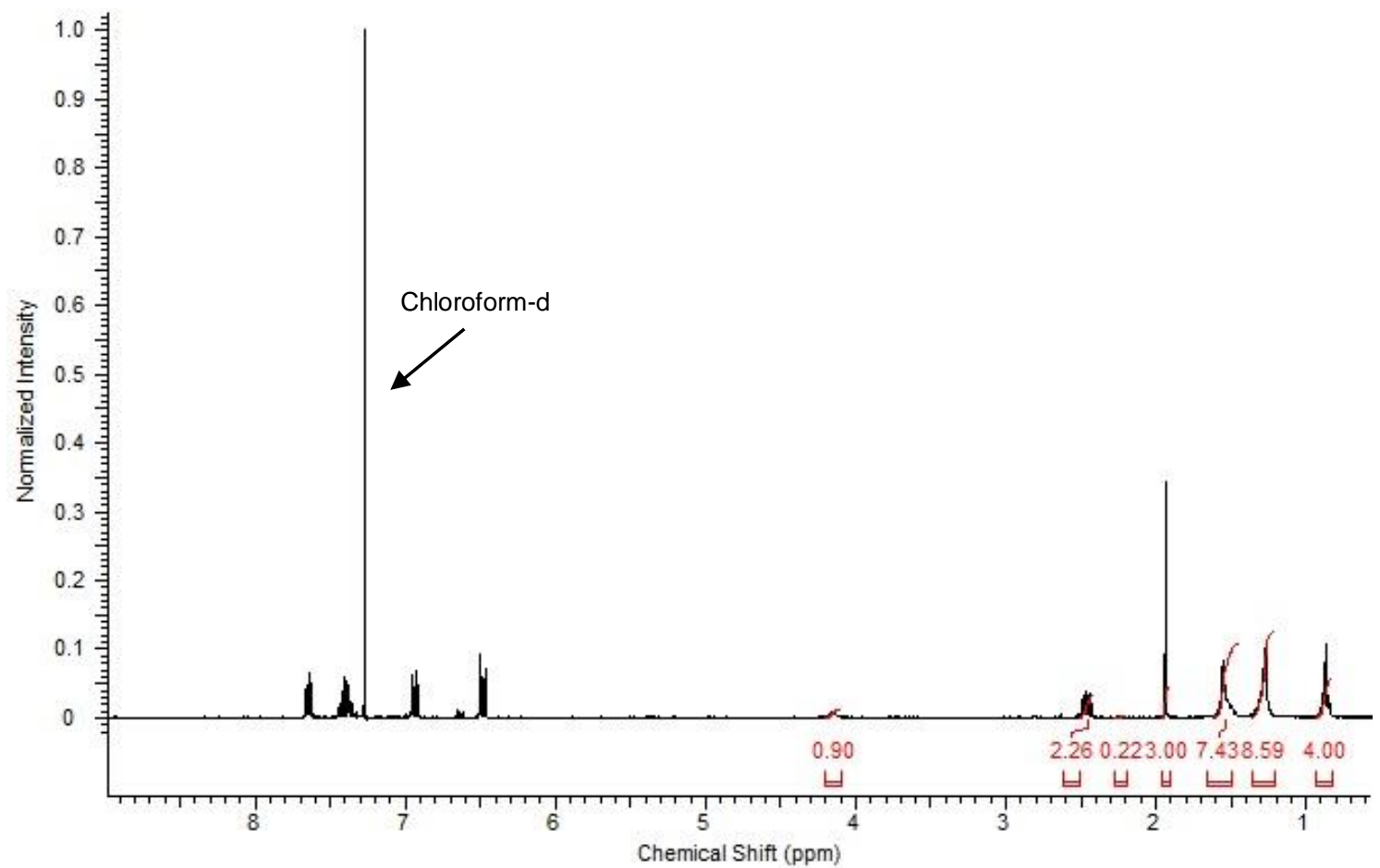

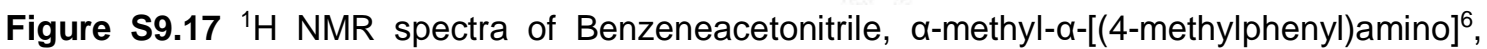
(Table 2, entry 19). 


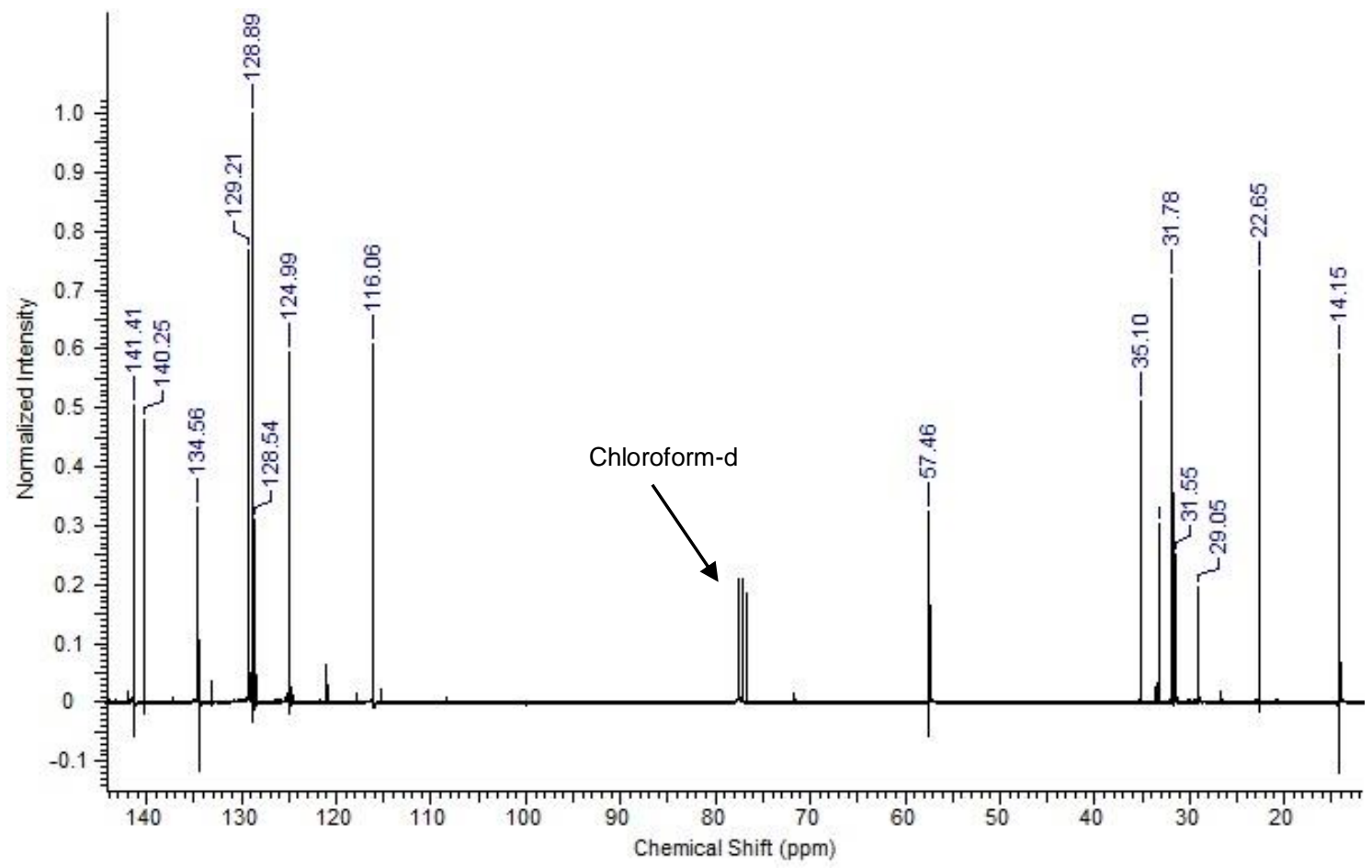

Figure S9.18 ${ }^{1} \mathrm{H}$ NMR spectra of Benzeneacetonitrile, $\alpha$-methyl- $\alpha-[(4-m e t h y l p h e n y l) a m i n o]^{6}$, (Table 2, entry 19).

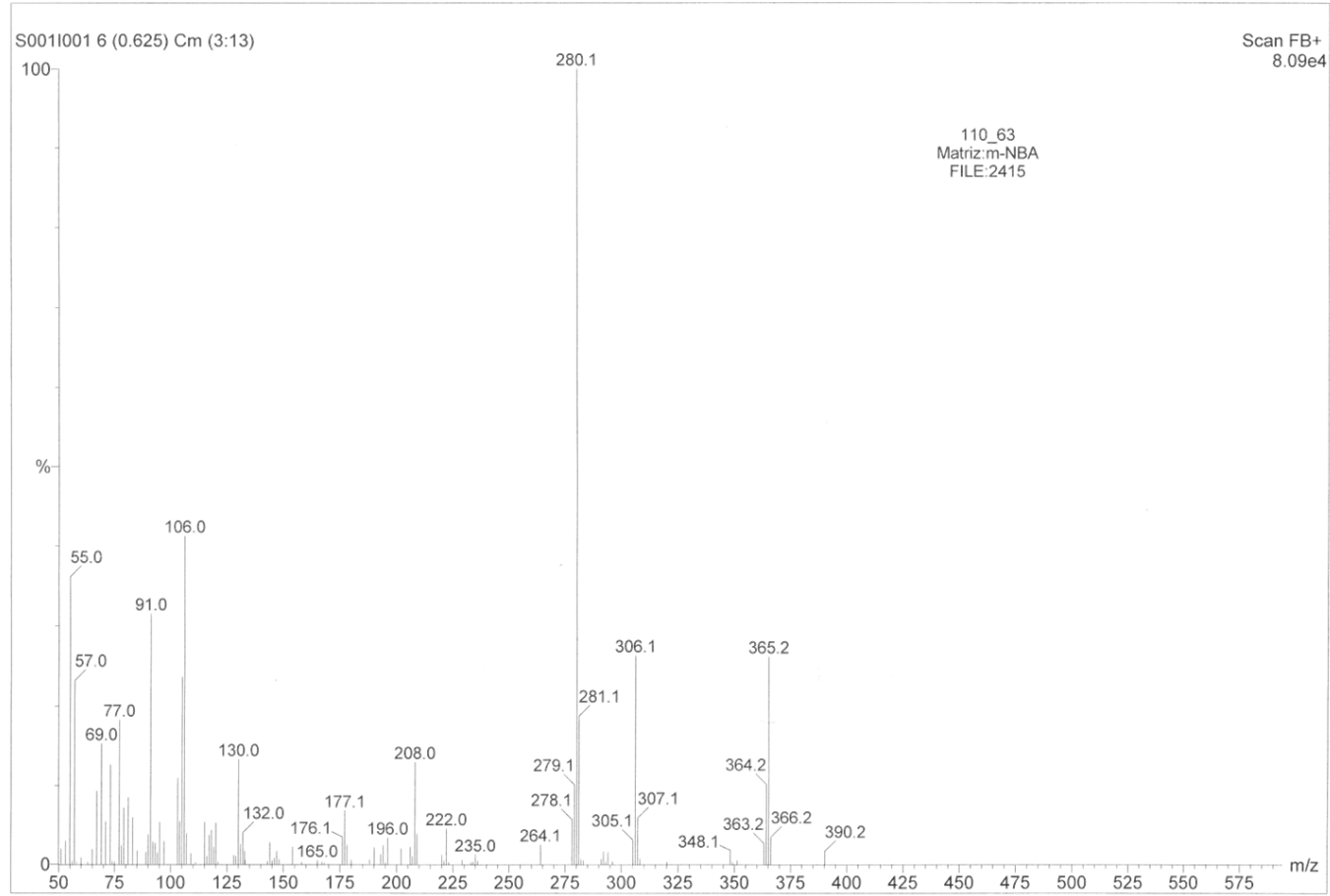

Figure S9.19 Mass spectra of Benzeneacetonitrile, $\alpha$-methyl- $\alpha-[(4-m e t h y l p h e n y l) a m i n o]^{6}$, (Table 2 , entry 19). 


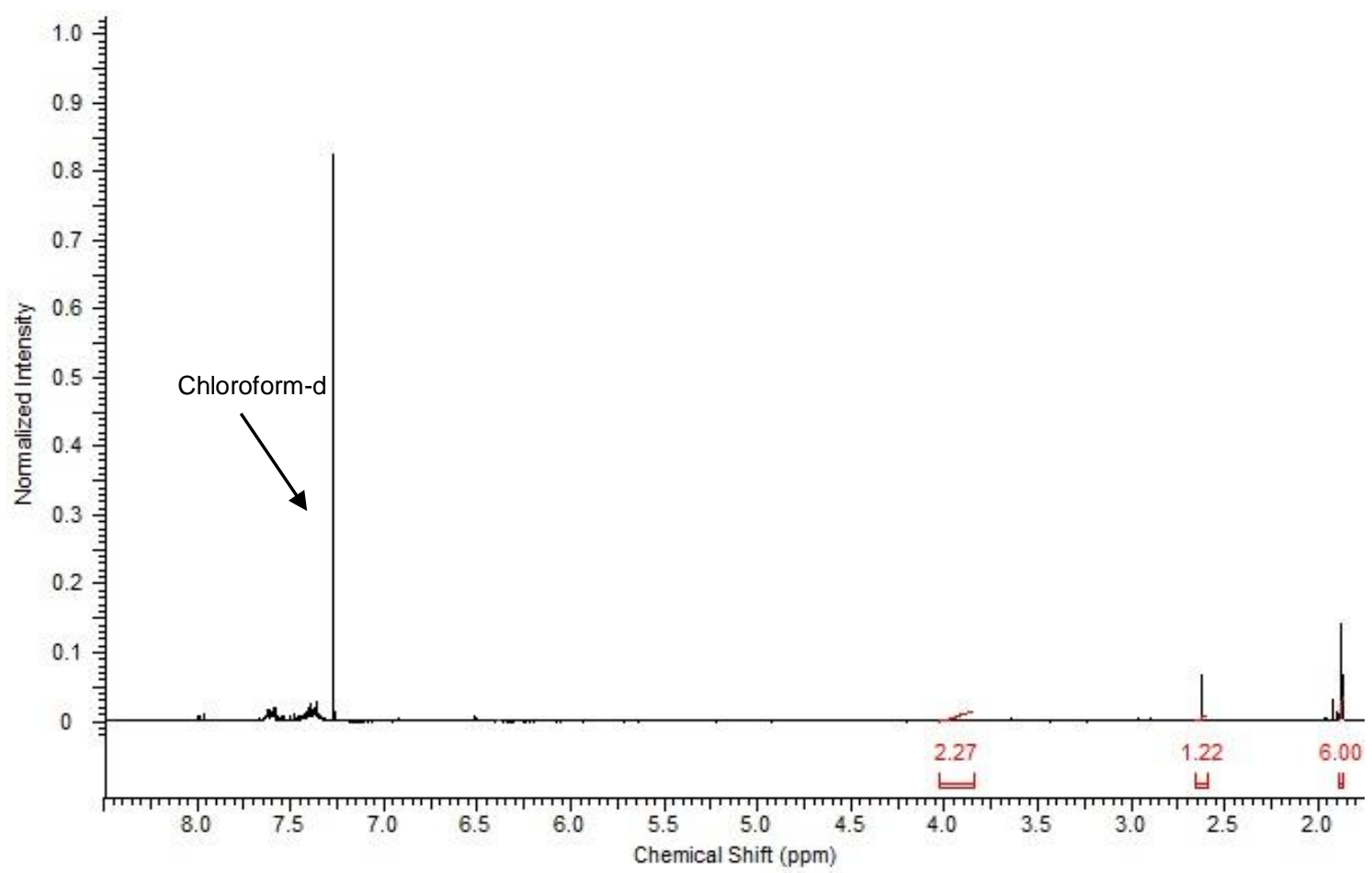

Figure S9.20 ${ }^{1} \mathrm{H}$ NMR spectra of Benzeneacetonitrile, $\alpha, \alpha^{\prime}-(1,4$-phenylenediimino)bis[a-methyl(9Cl)-;, , (Table 2, entry 19).

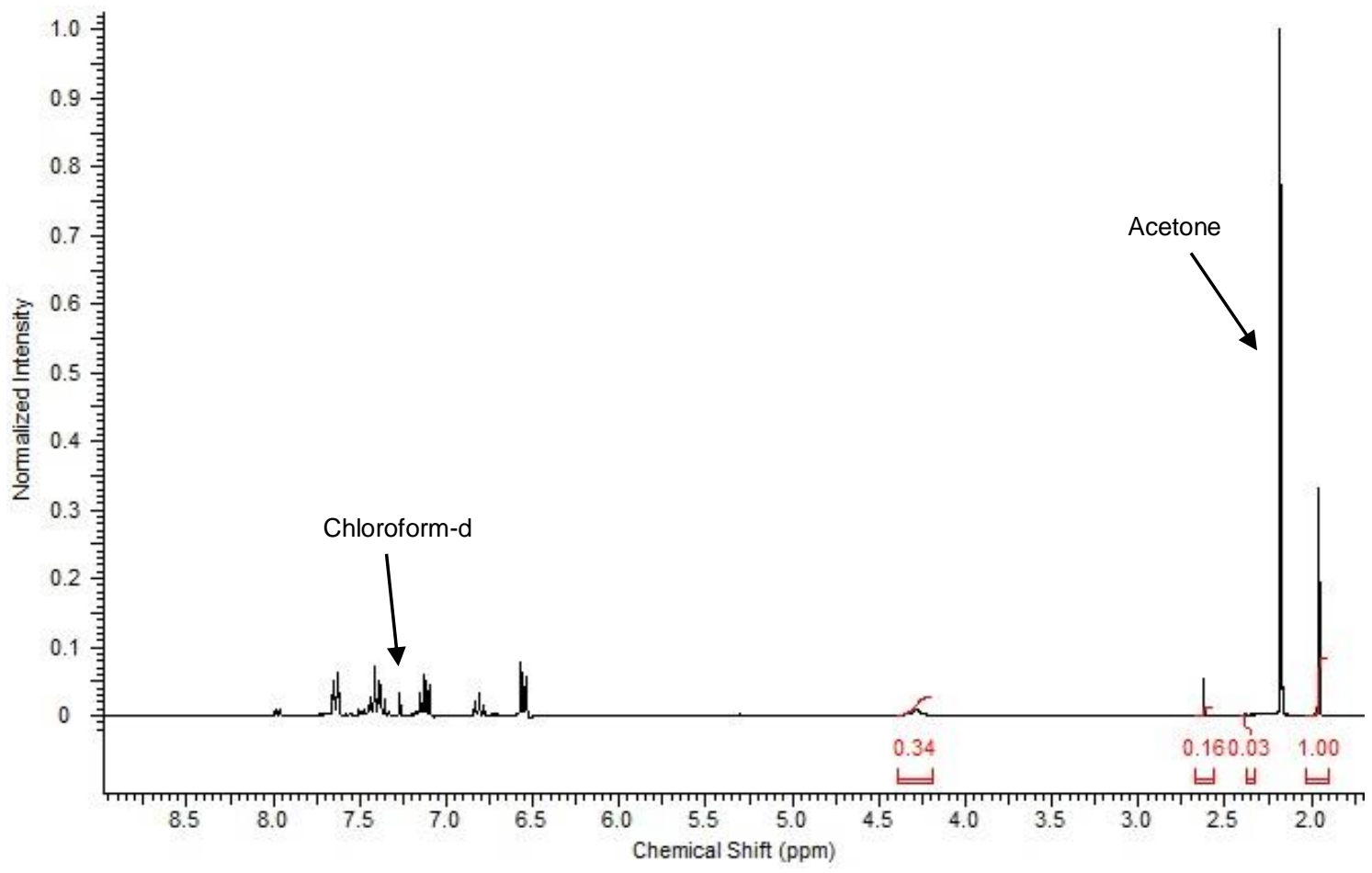

Figure S9.21 ${ }^{1} \mathrm{H}$ NMR spectra of 2-Phenyl-2-(phenylamino)propanenitrile. (Table S6, entry 1). 


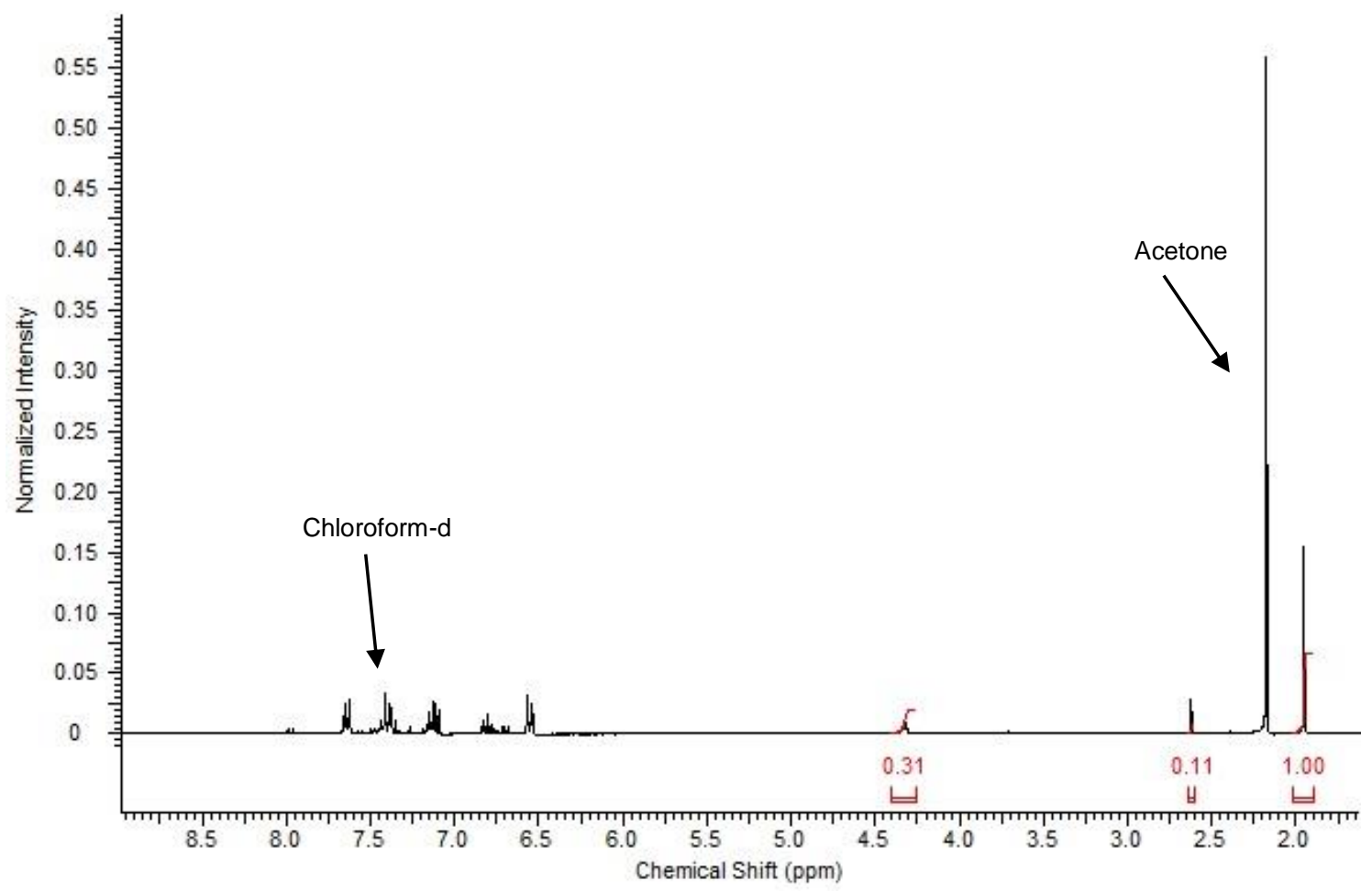

Figure S9.22 ${ }^{1} \mathrm{H}$ NMR spectra of 2-Phenyl-2-(phenylamino)propanenitrile. (Table S6, entry 2).

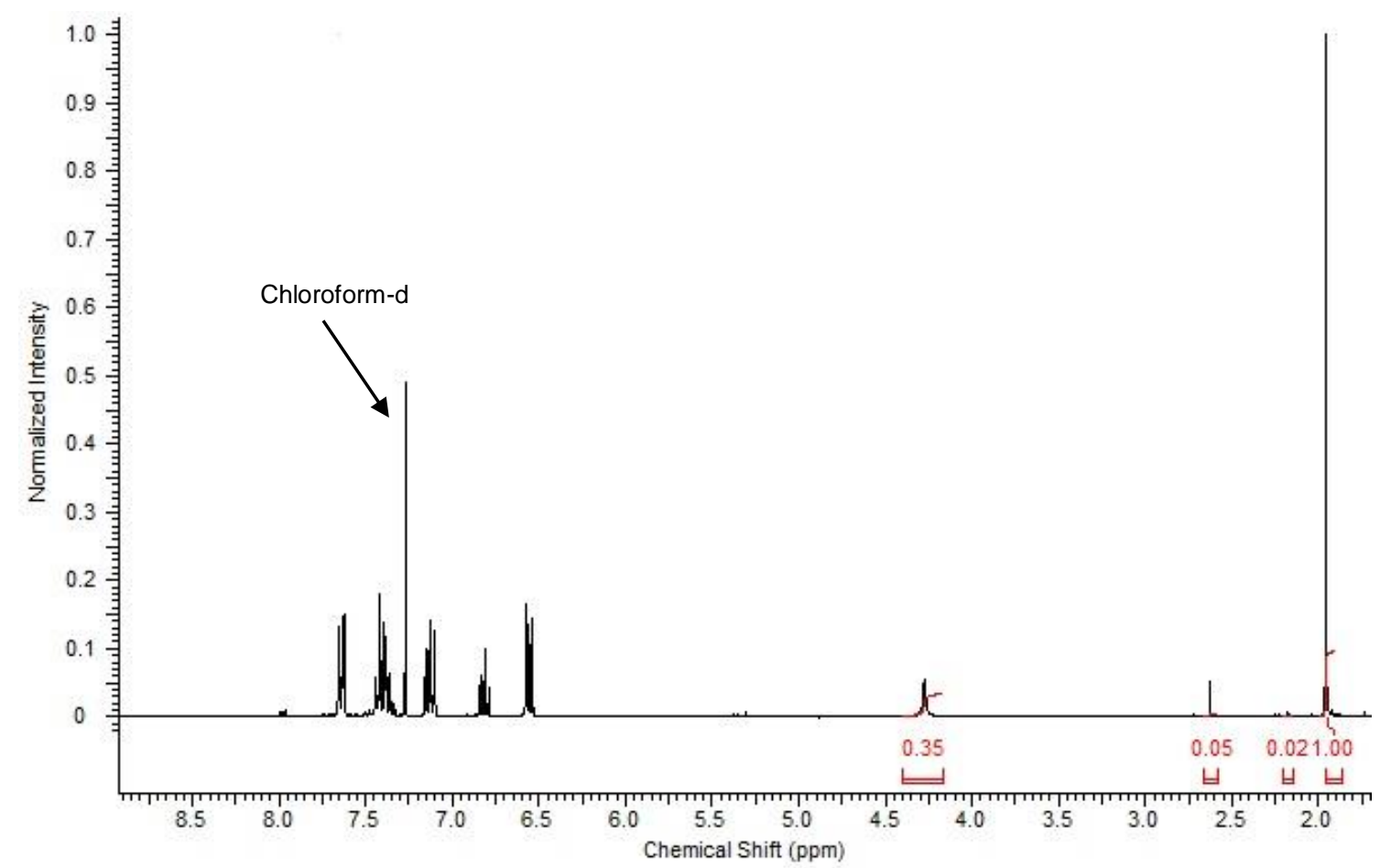

Figure S9.23 ${ }^{1} \mathrm{H}$ NMR spectra of 2-Phenyl-2-(phenylamino)propanenitrile. (Table S6, entry 4). 


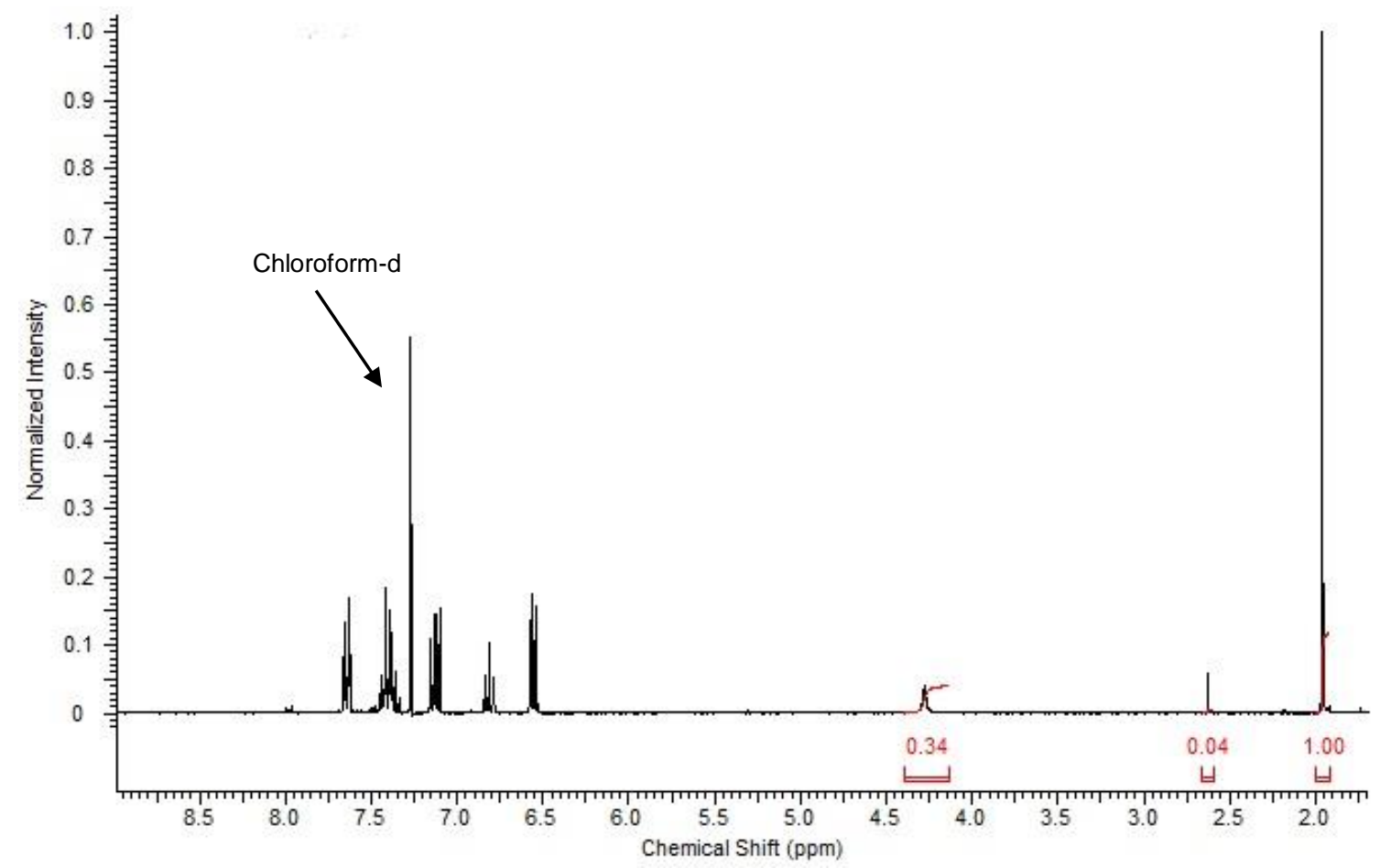

Figure S9.24 ${ }^{1} \mathrm{H}$ NMR spectra of 2-Phenyl-2-(phenylamino)propanenitrile. (Table S6, entry 5).

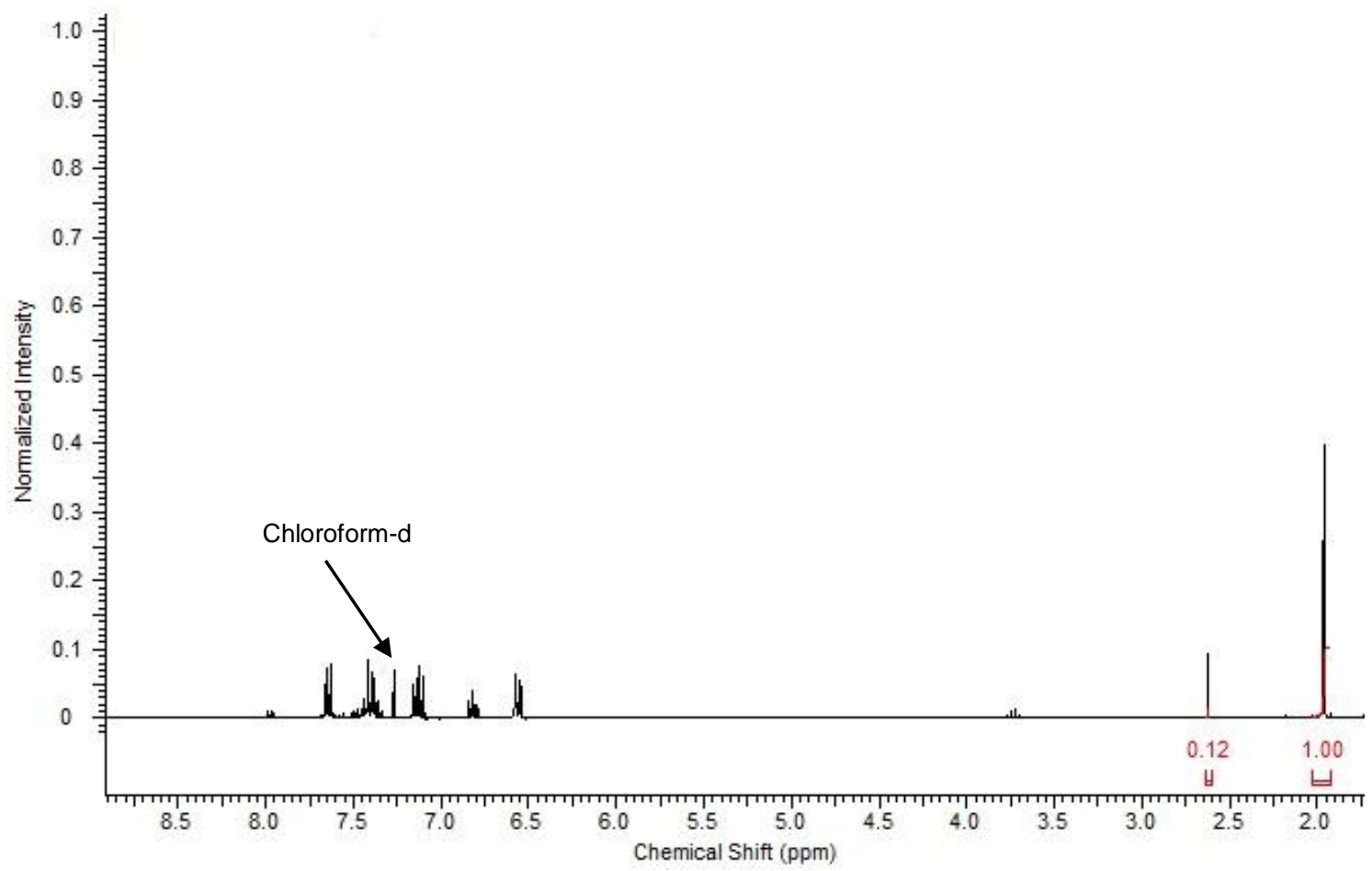

Figure S9.25 ${ }^{1} \mathrm{H}$ NMR spectra of 2-Phenyl-2-(phenylamino)propanenitrile. (Table S6, entry 6). 


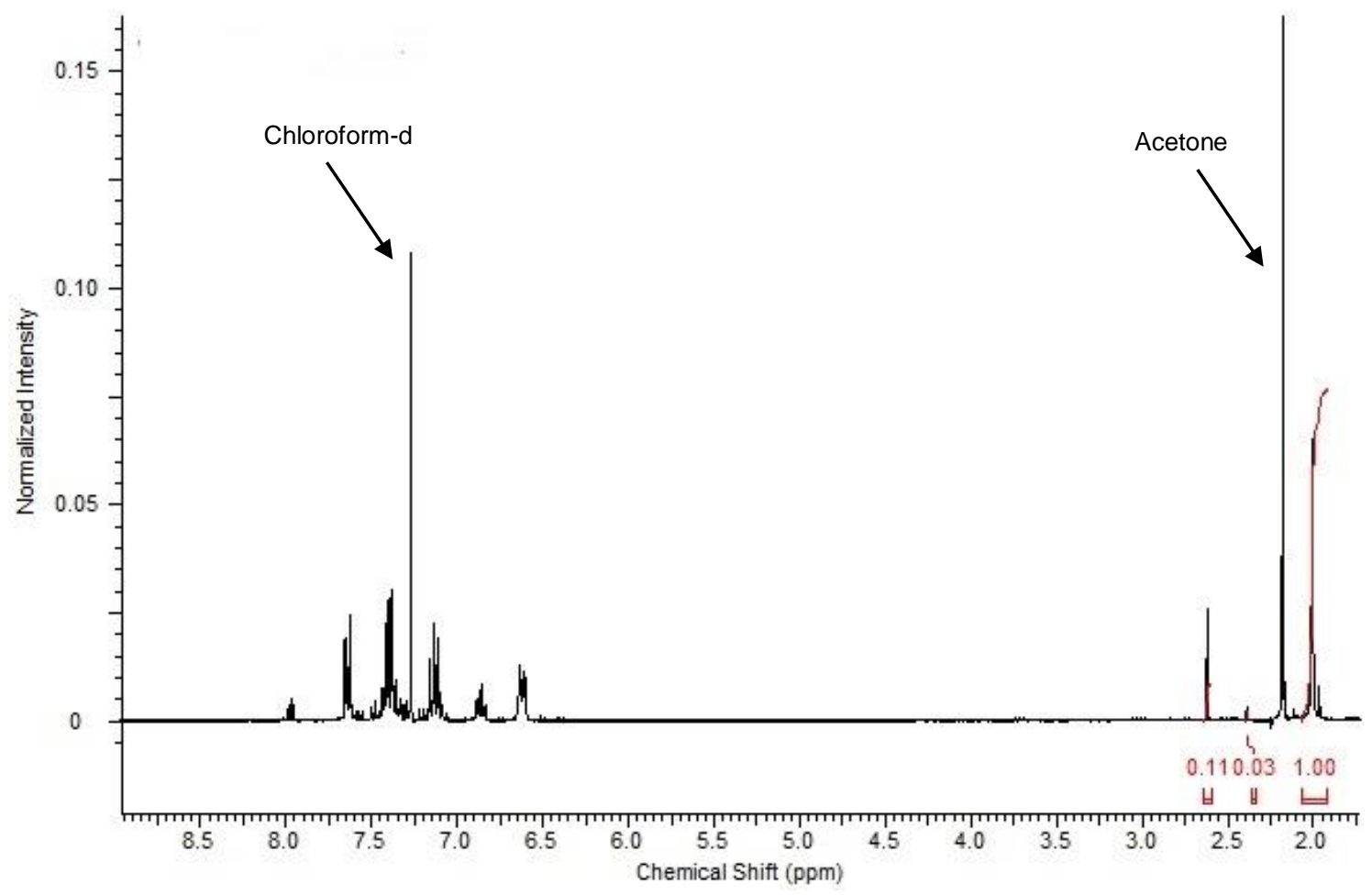

Figure S9.26 ${ }^{1} \mathrm{H}$ NMR spectra of 2-Phenyl-2-(phenylamino)propanenitrile. (Recycle Run 1).

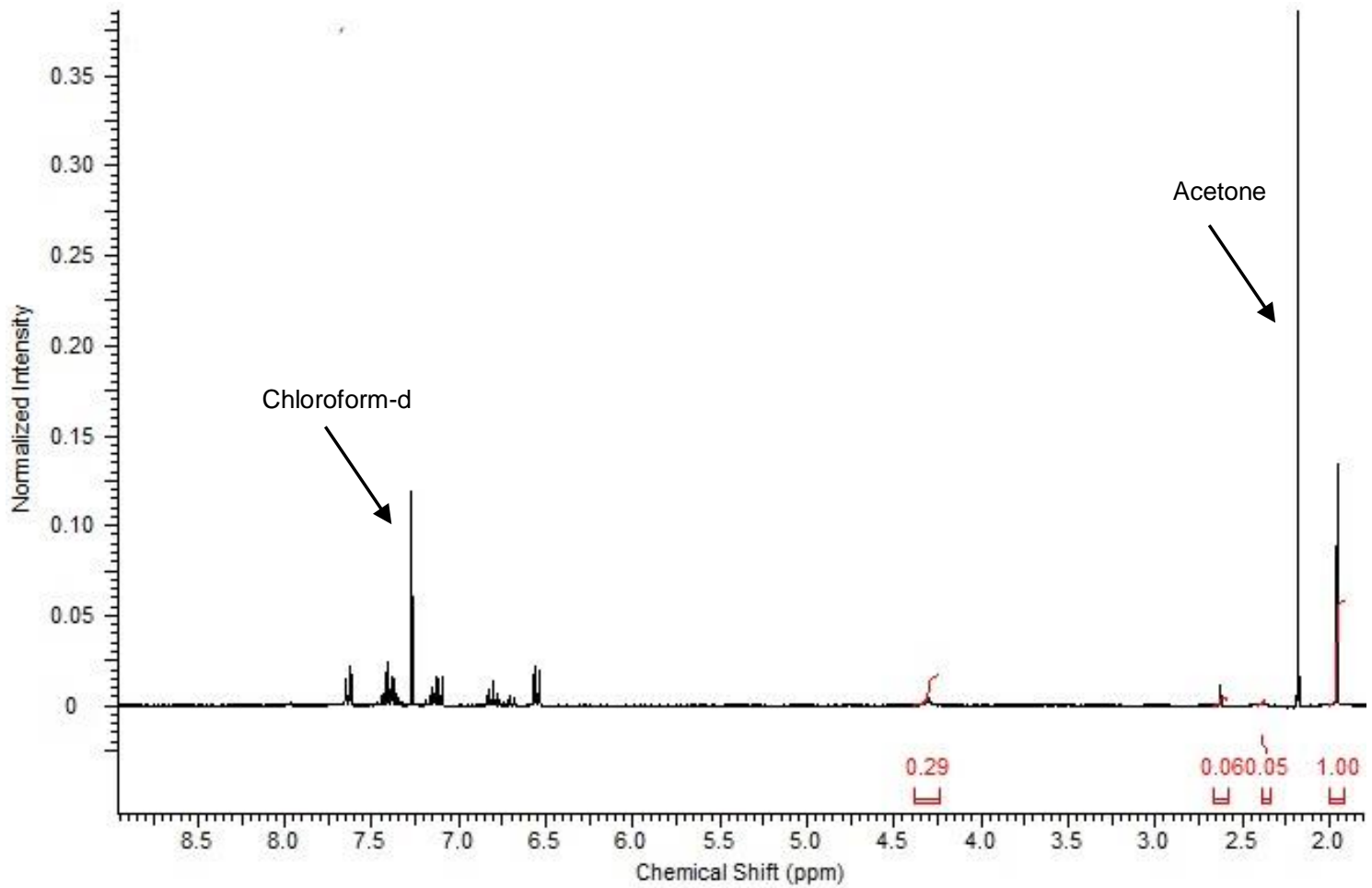

Figure S9.27 ${ }^{1} \mathrm{H}$ NMR spectra of 2-Phenyl-2-(phenylamino)propanenitrile. (Recycle Run 2). 


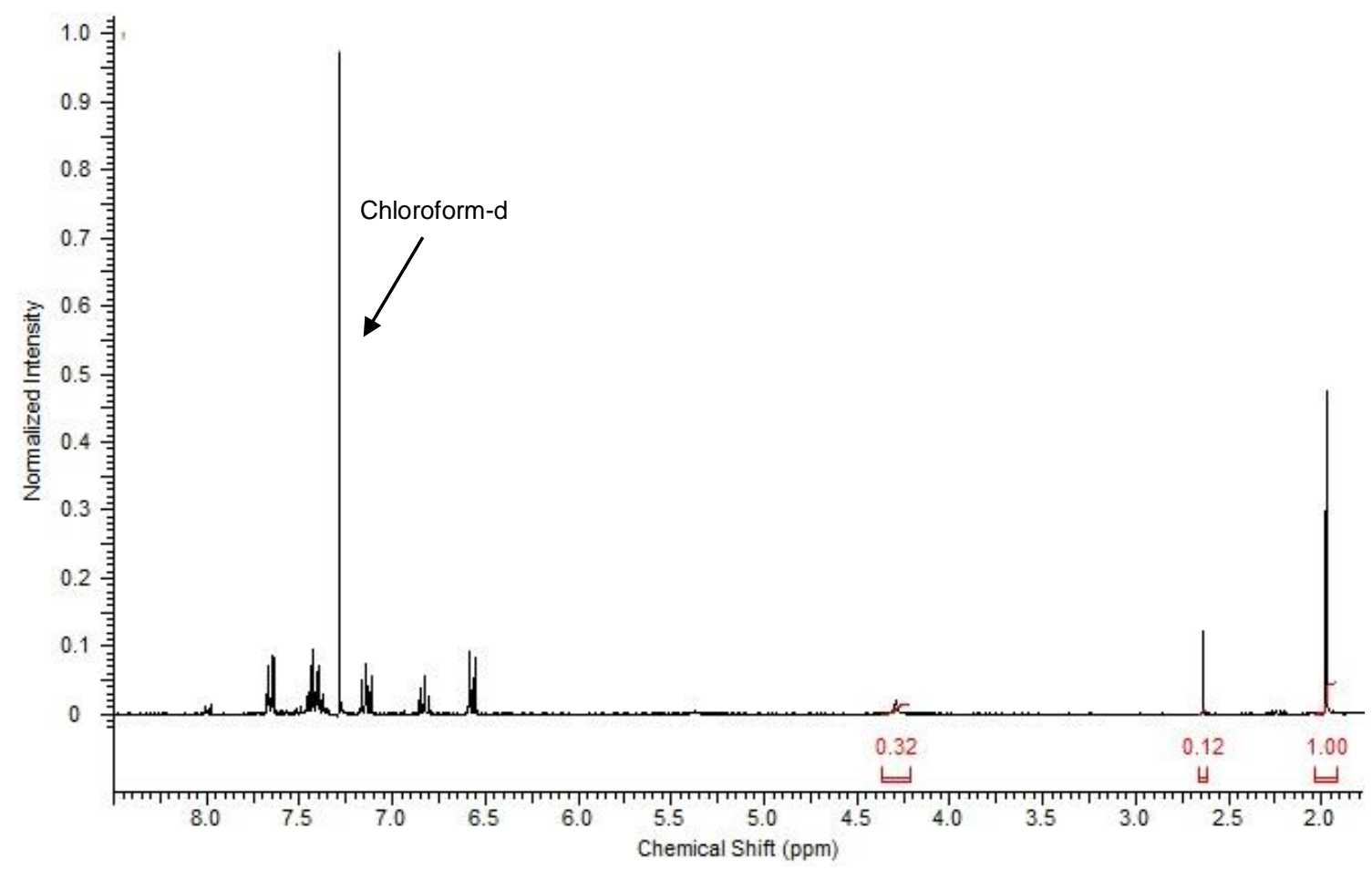

Figure S9.28 ${ }^{1} \mathrm{H}$ NMR spectra of 2-Phenyl-2-(phenylamino)propanenitrile. (Recycle Run 3).

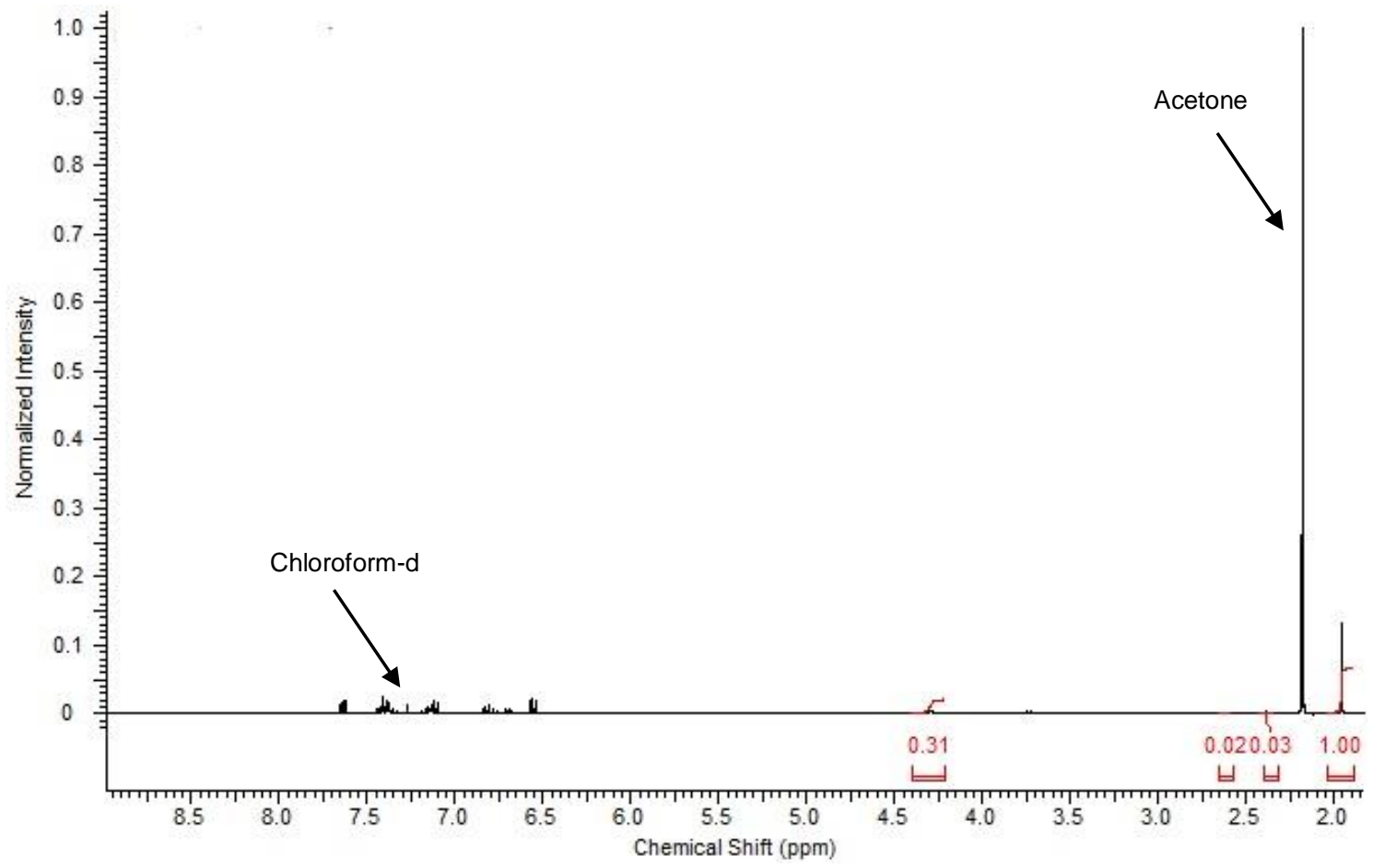

Figure S9.29 ${ }^{1} \mathrm{H}$ NMR spectra of 2-Phenyl-2-(phenylamino)propanenitrile. (Recycle Run 4). 


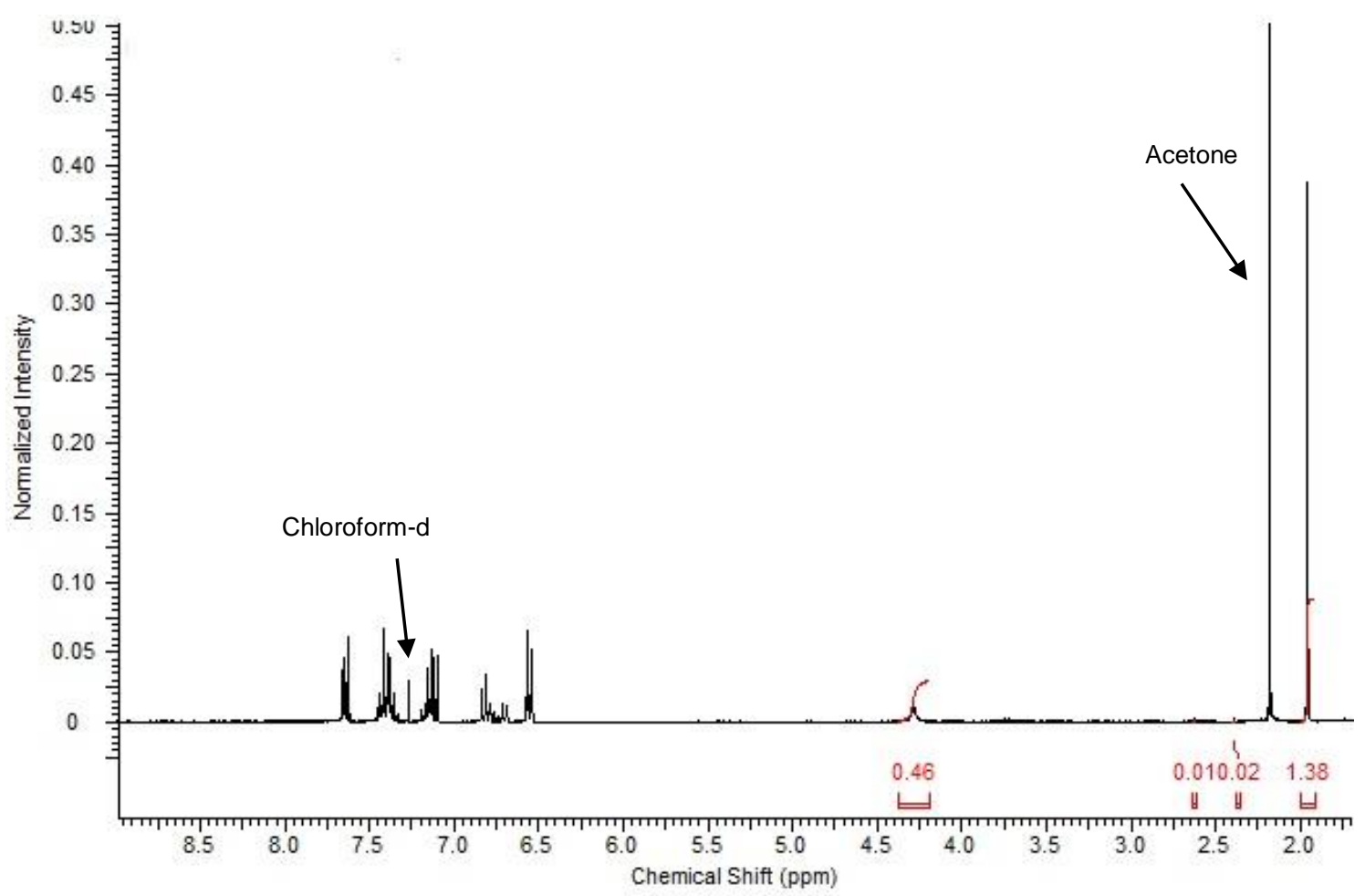

Figure S9.30 ${ }^{1} \mathrm{H}$ NMR spectra of 2-Phenyl-2-(phenylamino)propanenitrile. (Recycle Run 5).

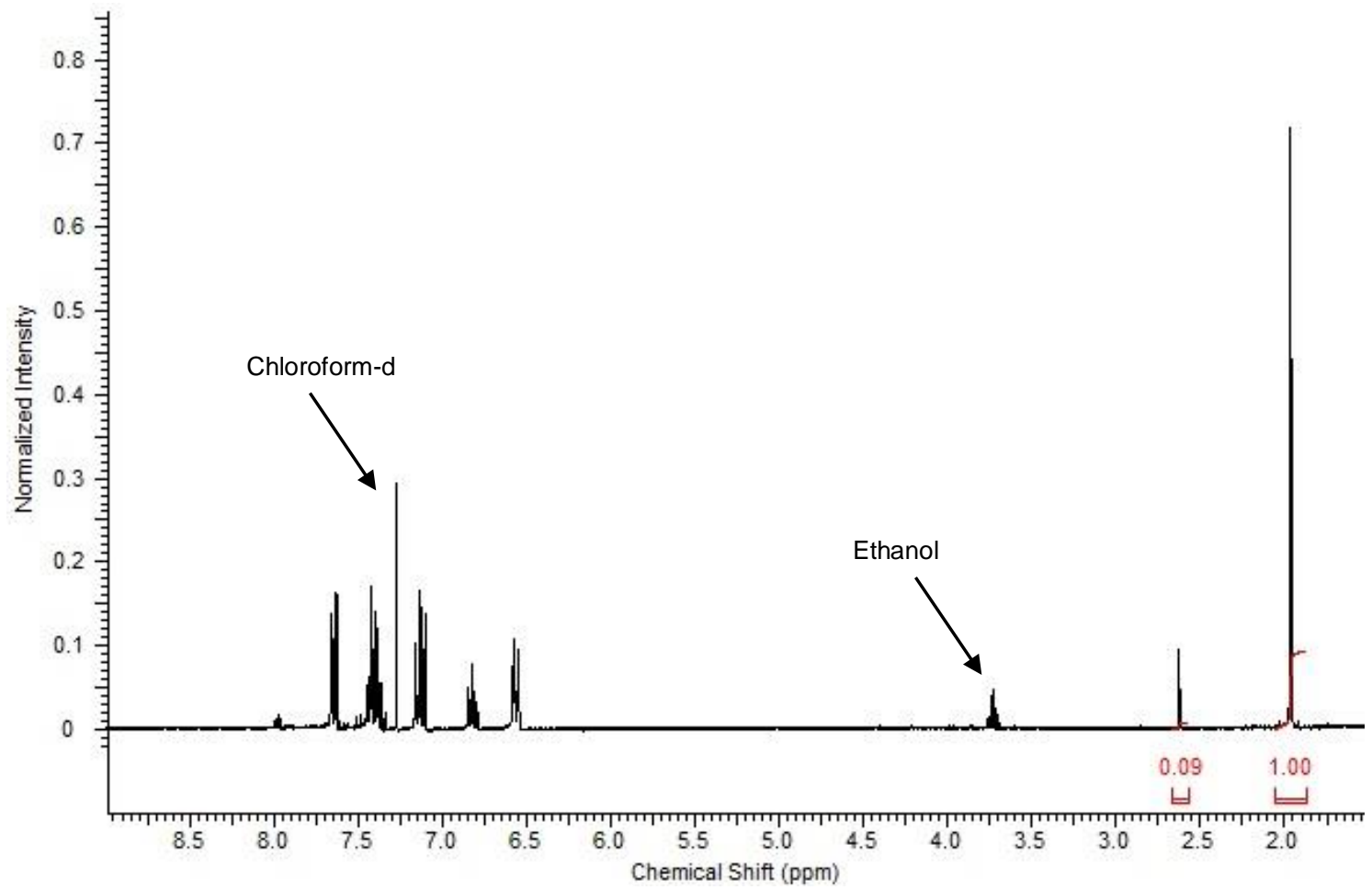

Figure S9.31 ${ }^{1} \mathrm{H}$ NMR spectra of 2-Phenyl-2-(phenylamino)propanenitrile. (Recycle Run 6). 


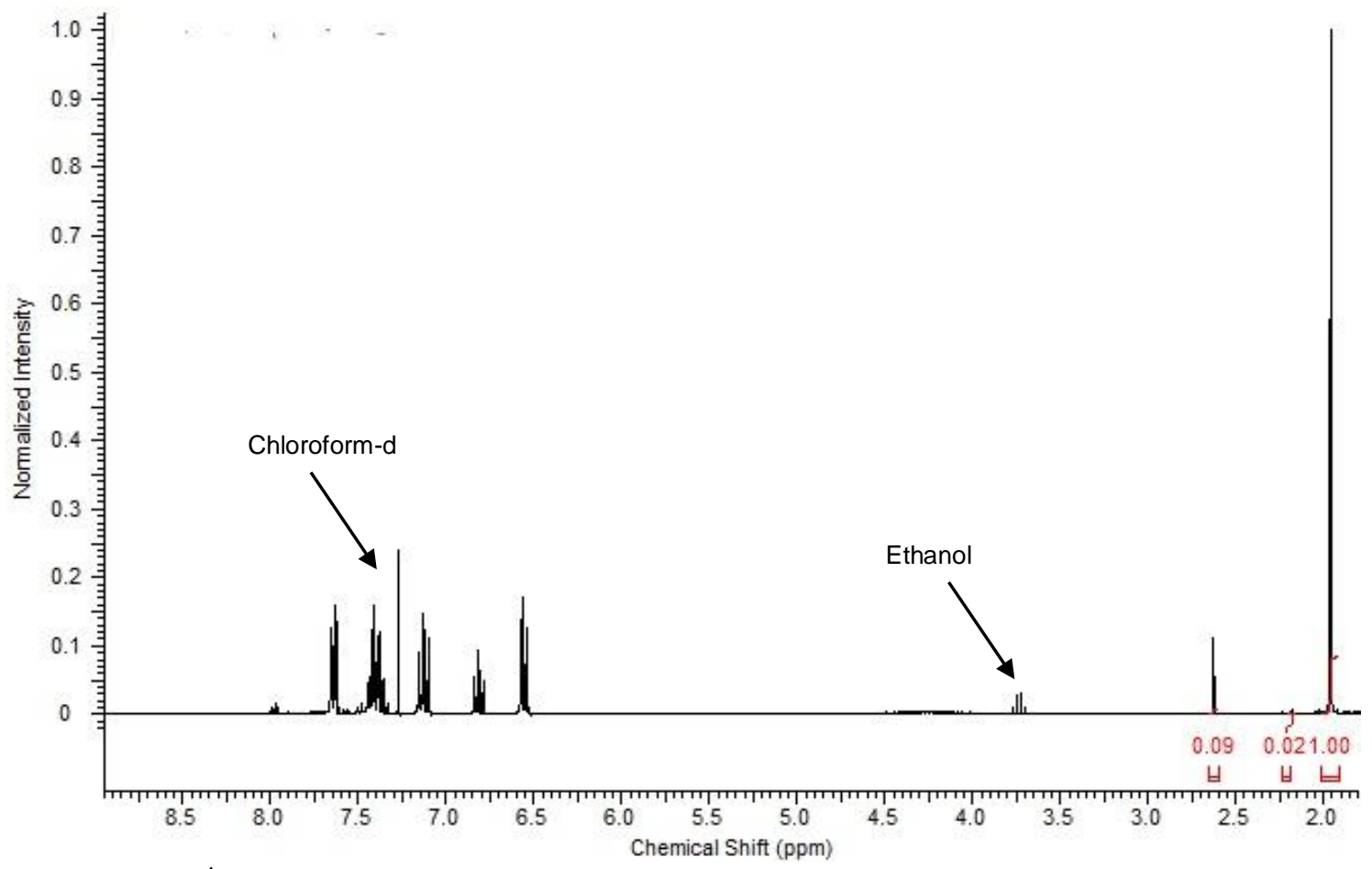

Figure S9.32 ${ }^{1} \mathrm{H}$ NMR spectra of 2-Phenyl-2-(phenylamino)propanenitrile. (Recycle Run 7).

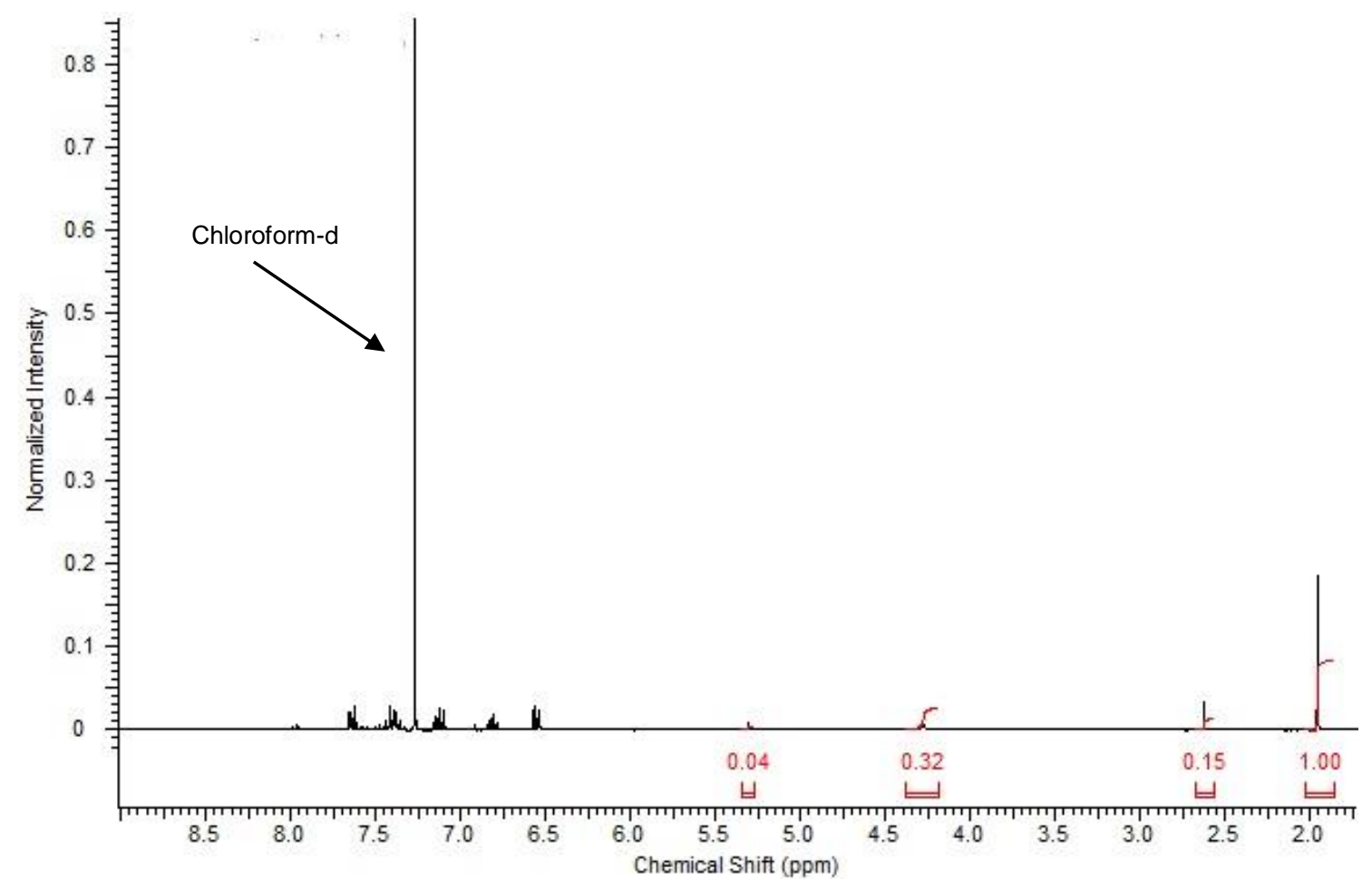

Figure S9.33 ${ }^{1} \mathrm{H}$ NMR spectra of 2-Phenyl-2-(phenylamino)propanenitrile. (Recycle Run 8). 


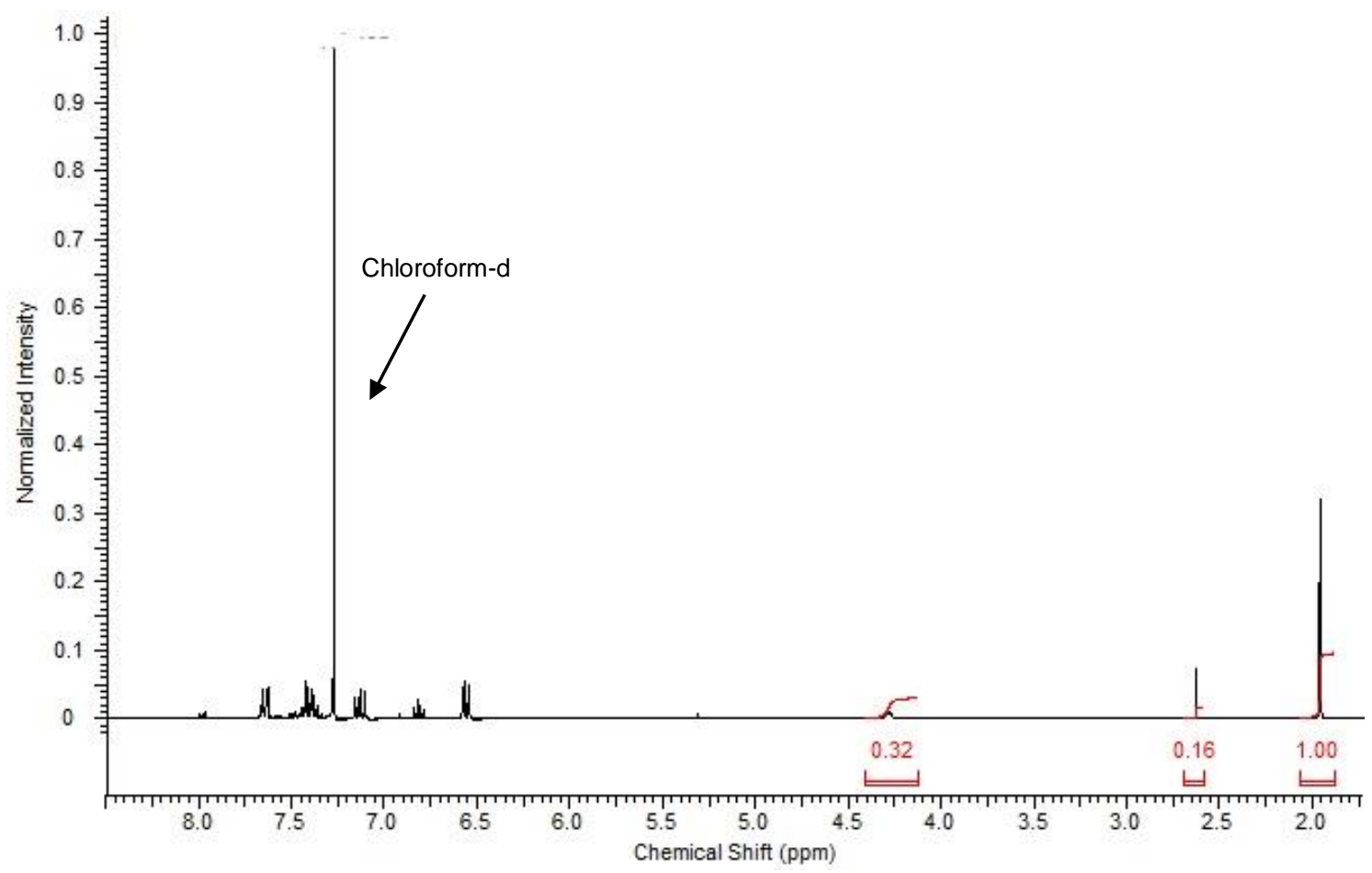

Figure S9.34 ${ }^{1} \mathrm{H}$ NMR spectra of 2-Phenyl-2-(phenylamino)propanenitrile. (Recycle Run 9).

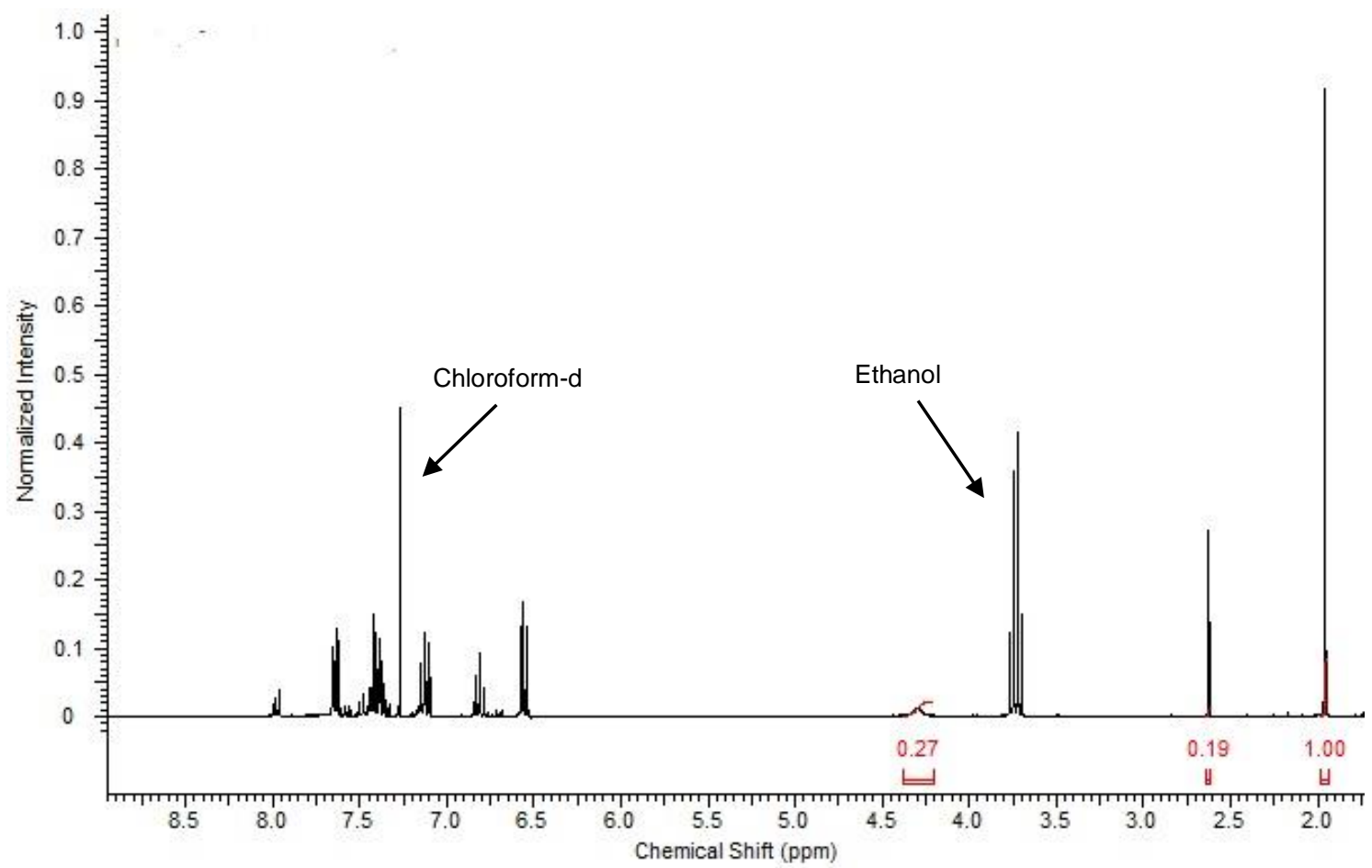

Figure S9.35 ${ }^{1} \mathrm{H}$ NMR spectra of 2-Phenyl-2-(phenylamino)propanenitrile. (Recycle Run 10). 
- S10. REFERENCES

(1) APEX2: Brucker-AXS: Madison, WI, 2006.

(2) Sheldrick, G. M. SHELXT-2014, 2013

(3) Dolomanov, O. V.; Bourhis, L. J.; Gildea, R. J.; Howard, J. A. K.; Puschmann, H. J. Appl. Crystallogr. 2009, 42, 339-341.

(4) Sheldrick, G. M. Acta Crystallogr. Sect. A 2008, 64, 112-122

(5) Inorganic Crystal Structure Database, ICSD.

(6) Barbero, M.; Cadamuro, S.; Dughera, S.; Ghigo, G. Organic \& Biomolecular Chemistry 2012, 10 (20), 4058-4068.

(7) Karimi, B.; Zareyee, D. J. Mater. Chem. 2009, 19 (45), 8665-8670.

(8) Bychinskaya, I.; Marinez, E. R.; Matthew, T.; Olah, G. Catalysis Letters. 2013, 143 (4), 303312.

(9) Prakash, G. K. S.; Mathew, T.; Olah, G. A. Accounts Chem. Res. 2012, 45 (4), 565-577. (10) Zhang, G.-W.; Zheng, D.-H.; Nie, J.; Wang, T.; Ma, J.-A. Organic \& Biomolecular Chemistry 2010, 8 (6), 1399-1405.

(11) Hamachi, Y.; Katano, M.; Ogiwara, Y.; Sakai N. Organic Letters 201618 (7), 1634-1637. (12) Xia, J.; Xu, J. N.; Fan, Y.; Song, T. Wang, Li.; Zheng, J. Y. Inorg. Chem. 2014, 53 (19), 10024-10026

(13) Aguirre-Díaz, L. M.; Gándara, F.; Iglesias, M.; Snejko, N.; Gutiérrez-Puebla, E.; Monge, M. Á. Journal of the American Chemical Society 2015, 137 (19), 6132-6135.

(14) Shah, A. K.; Khan, N. H.; Sethia, G.; Saravanan, S.; Kureshy, R. I.; Abdi, S. H. R.; Bajaj, H. C. Applied Catalysis A: General 2012, 420, 22-30.

(15) Pathare, S. P.; Akamanchi, K. G. Tetrahedron Letters 2012, 53 (7), 871-875. 